\title{
The tangential $k$-Cauchy-Fueter complexes and Hartogs' phenomenon over the right quaternionic Heisenberg group
}

\author{
Yun $\mathrm{Shi}^{1} \cdot$ Wei Wang ${ }^{2}$
}

Received: 11 September 2018 / Accepted: 17 August 2019 / Published online: 26 August 2019

(c) Fondazione Annali di Matematica Pura ed Applicata and Springer-Verlag GmbH Germany, part of Springer Nature 2019

\begin{abstract}
We construct the tangential $k$-Cauchy-Fueter complexes on the right quaternionic Heisenberg group, as the quaternionic counterpart of $\bar{\partial}_{b}$-complex on the Heisenberg group in the theory of several complex variables. We can use the $L^{2}$ estimate to solve the nonhomogeneous tangential $k$-Cauchy-Fueter equation under the compatibility condition over this group modulo a lattice. This solution has an important vanishing property when the group is higher dimensional. It allows us to prove the Hartogs' extension phenomenon for $k$-CF functions, which are the quaternionic counterpart of CR functions.
\end{abstract}

Keywords The tangential $k$-Cauchy-Fueter complex · Hartogs' phenomenon · The right quaternionic Heisenberg group · The nonhomogeneous equation under the compatibility condition - The $L^{2}$ estimate

\section{Introduction}

The $\bar{\partial}$-complex plays an important role in the theory of several complex variables since many important results for holomorphic functions can be obtained by solving nonhomogeneous $\bar{\partial}$-equation. We obtain $\bar{\partial}_{b}$-complex when it is restricted to a CR submanifold, and many important results for CR functions can also be obtained by solving $\bar{\partial}_{b}$-equation. In general, for a differential complex, there is an abstract way to obtain a boundary complex restricted to a submanifold, which is written down in terms of quotient sheafs (cf., e.g., [3,4,25]).

The first author is partially supported by National Nature Science Foundation in China (Nos. 11801508, 11571305); The second author is partially supported by National Nature Science Foundation in China (No. 11571305).

$凶 \quad$ Wei Wang

wwang@zju.edu.cn

Yun Shi

hzxjhs1987@163.com

1 Department of Mathematics, Zhejiang University of Science and Technology, Hangzhou 310023, China

2 Department of Mathematics, Zhejiang University, Hangzhou 310027, China 
In quaternionic analysis, we now know the $k$-Cauchy-Fueter complexes explicitly (cf. $[1,5,8,9,11,30,35,41]$ and references therein), which are used to show several interesting properties of $k$-regular functions (cf. [12,35,40,42] and references therein). When restricted to a quadratic hypersurface in $\mathbb{H}^{n+1}$, we have the tangential $k$-Cauchy-Fueter operators and $k$-CF functions (cf. [39] for $k=1, n=2$ ), corresponding to $\bar{\partial}_{b}$ and CR functions over a CR manifold. In this paper, we will consider their restriction to a model quadratic hypersurface

$$
\mathcal{S}:=\left\{\left(q^{\prime}, q_{n+1}\right) \in \mathbb{H}^{n} \times \mathbb{H}: \rho\left(q^{\prime}, q_{n+1}\right)=0\right\}
$$

in $\mathbb{H}^{n+1}$, where

$$
\rho\left(q^{\prime}, q_{n+1}\right):=\operatorname{Re} q_{n+1}-\phi\left(q^{\prime}\right), \quad \phi\left(q^{\prime}\right):=\sum_{l=0}^{n-1}\left(-3 x_{4 l+1}^{2}+x_{4 l+2}^{2}+x_{4 l+3}^{2}+x_{4 l+4}^{2}\right) .
$$

Here, we write $q^{\prime}=\left(\ldots, q_{l}, \ldots\right), q_{l}=x_{4 l+1}+\mathbf{i} x_{4 l+2}+\mathbf{j} x_{4 l+3}+\mathbf{k} x_{4 l+4}$. This hypersurface has the structure of the right quaternionic Heisenberg group $\mathscr{H}=\mathbb{H}^{n} \times \operatorname{Im} \mathbb{H}$ with the multiplication given by

$$
(x, t) \cdot(y, s)=(x+y, t+s+2 \operatorname{Im}(x \bar{y})),
$$

where $x, y \in \mathbb{H}^{n}$ and $t, s \in \operatorname{Im} \mathbb{H}$. We construct a family of differential complexes on $\mathscr{H}$, the tangential $k$-Cauchy-Fueter complexes, given by

$$
0 \rightarrow C^{\infty}\left(\Omega, \mathscr{V}_{0}\right) \stackrel{\mathscr{D}_{0}}{\longrightarrow} C^{\infty}\left(\Omega, \mathscr{V}_{1}\right) \stackrel{\mathscr{D}_{1}}{\longrightarrow} C^{\infty}\left(\Omega, \mathscr{V}_{2}\right) \rightarrow \cdots \stackrel{\mathscr{D}_{2 n-2}}{\longrightarrow} C^{\infty}\left(\Omega, \mathscr{V}_{2 n-1}\right) \rightarrow 0,
$$

for a domain $\Omega$ in $\mathscr{H}$, where

$$
\begin{aligned}
& \mathscr{V}_{j}:=\odot^{k-j} \mathbb{C}^{2} \otimes \wedge^{j} \mathbb{C}^{2 n}, \quad j=0,1, \ldots, k, \\
& \mathscr{V}_{j}:=\odot^{j-k-1} \mathbb{C}^{2} \otimes \wedge^{j+1} \mathbb{C}^{2 n}, \quad j=k+1, \ldots, 2 n-1,
\end{aligned}
$$

for fixed $k=0,1, \ldots$, and $\odot^{p} \mathbb{C}^{2}$ is the $p$ th symmetric power of $\mathbb{C}^{2}$. They are the quaternionic counterpart of $\bar{\partial}_{b}$-complex over the Heisenberg group in the theory of several complex variables. They have the same form as the $k$-Cauchy-Fueter complexes on $\mathbb{H}^{n}$ (cf. Remark 2.1 ), but $\mathscr{D}_{j}$ 's are given in terms of left invariant vector fields (2.23) (2.26) (2.27), which are differential operators of variable coefficients. So we cannot use the computational algebraic method in [12] to construct these complexes. This family of complexes are natural in the sense that they can be viewed as the restriction to the hypersurface $\mathcal{S}$ of complexes on $\mathbb{H}^{n+1}$, but not natural in the sense that they are not invariant under the conformal transformation group $\operatorname{Sp}(n+1,1)$ of $\mathscr{H}$ (cf. Sect. 2.5).

$\mathscr{D}_{0}$ in (1.4) is called the tangential $k$-Cauchy-Fueter operator. A $\odot^{k} \mathbb{C}^{2}$-valued distribution $f$ on $\Omega$ is called $k-C F$ if $\mathscr{D}_{0} f=0$ in the sense of distributions. The space of all $k$-CF functions on $\Omega$ is denoted by $\mathcal{A}_{k}(\Omega)$. A 1-CF function is also called anti-CRF function in $[18,19]$. Such functions play an important role in the study of pseudo-Einstein equation over the quaternionic Heisenberg group [19].

On the other hand, when the hypersurface is the boundary of the Siegel upper half space, i.e., the defining function in (1.1) is given by

$$
\rho=\operatorname{Re} q_{n+1}-\left|q^{\prime}\right|^{2},
$$


the corresponding group is the left quaternionic Heisenberg group $\widetilde{\mathscr{H}}:=\mathbb{H}^{n} \times \operatorname{Im} \mathbb{H}$ with the multiplication given by

$$
(x, t) \cdot(y, s)=(x+y, t+s+2 \operatorname{Im}(\bar{x} y)) .
$$

We already know the tangential $k$-Cauchy-Fueter complex (cf. [37, Theorem 1.0.1]) on the left quaternionic Heisenberg group by using the twistor method (see also [6,27] for constructing complexes by this method). But in this case, $\wedge^{j} \mathbb{C}^{2 n}$ in (1.5) must be replaced by the irreducible representation of $\mathfrak{s p}(2 n, \mathbb{C})$ with the highest weight to be the $j$ th fundamental weight (cf. Sect. 2.5). Since it is more complicated than the right case, we only consider the right quaternionic Heisenberg group in this paper. We see that when restricted to different submanifolds, we get different differential complexes. This is a new phenomenon compared to several complex variables, where expressions of $\bar{\partial}_{b}$-complex for different CR submanifolds are the same. It is an interesting problem to write down explicitly the tangential $k$-CauchyFueter complexes for all quadratic hypersurfaces in $\mathbb{H}^{n+1}$ (cf. [39] for such hypersurfaces).

In this paper, we prove Hartogs' phenomenon for $k$-CF functions over right quaternionic Heisenberg group.

Theorem 1.1 Let $\Omega$ be a bounded open set in the right quaternionic Heisenberg group $\mathscr{H}$ with $\operatorname{dim} \mathscr{H} \geq 19$, and let $K$ be a compact subset of $\Omega$ such that $\Omega \backslash K$ is connected. Then, for each $u \in \mathcal{A}_{k}(\Omega \backslash K), k=2,3, \ldots$, we can find $U \in \mathcal{A}_{k}(\Omega)$ such that $U=u$ in $\Omega \backslash K$.

The restriction of $\operatorname{dim} \mathscr{H}$ and $k$ in this theorem comes from the technical difficulty to establish the $L^{2}$ estimate in the remaining cases. A form of Hartogs' phenomenon was proved for many elliptic differential systems (cf. [12,26] and references therein). Notably, in our case $\mathscr{D}_{0}$ as a matrix-valued horizontal vector field is not an elliptic system, and (1.4) is not an elliptic complex. This is because symbols of $\mathscr{D}_{j}$ 's vanish at the cotangent vectors annihilating horizontal vector fields.

In the complex case, we have deep Hartogs-Bochner effect for CR functions on CR submanifolds, which are usually proved by using integral representation formulae (cf. [15,23,29] and references therein for further development of this effect). But in the quaternionic case, the integral representation formulae are not sufficiently developed, and only Bochner-Martinellitype formulae are known (cf. [34,35]). As in the theory of several complex variables, the formulae with Bochner-Martinelli- type kernels are not good enough to prove the extension phenomenon.

Given a differential complex, it is a fundamental problem to investigate its cohomology group or its Poincaré lemma over a domain (cf., e.g., [7,16]). In particular, we hope to solve the nonhomogeneous tangential $k$-Cauchy-Fueter equation

$$
\mathscr{D}_{0} u=f,
$$

for $f \in L^{2}\left(\mathscr{H}, \mathscr{V}_{1}\right)$, under the compatibility condition

$$
\mathscr{D}_{1} f=0,
$$

i.e., $f$ is $\mathscr{D}_{1}$-closed. If we can find compactly supported solution of (1.7)-(1.8) when $f$ is compactly supported, it is a standard procedure to derive Hartogs' phenomenon (cf., e.g., $[17,35])$. One way to solve $(1.7)-(1.8)$ is to consider the associated Hodge-Laplacian

$$
\square_{1}=\mathscr{D}_{0} \mathscr{D}_{0}^{*}+\mathscr{D}_{1}^{*} \mathscr{D}_{1}: L^{2}\left(\mathscr{H}, \mathscr{V}_{1}\right) \rightarrow L^{2}\left(\mathscr{H}, \mathscr{V}_{1}\right) .
$$

By identifying $\mathscr{V}_{1}=\odot^{k-1} \mathbb{C}^{2} \otimes \mathbb{C}^{2 n}$ with $\mathbb{C}^{2 n k}$, we can see that $\square_{1}$ is a $(2 k n) \times(2 k n)$ matrix-valued differential operator of the second order, which is not diagonal (cf. Appendix 
for the expression in the case $n=2, k=2$ ). So it is not easy to verify the subellipticity of $\square_{1}$ and find its fundamental solution, while in the complex case, the Hodge-Laplacian associated with $\bar{\partial}_{b}$-complex is diagonal and it is easy to find its fundamental solution (cf. [13]).

By using the $L^{2}$ method, we establish the following estimate: when $\operatorname{dim} \mathscr{H} \geq 19$, there exists some constant $c>0$ such that

$$
\left\|\mathscr{D}_{0}^{*} f\right\|^{2}+\left\|\mathscr{D}_{1} f\right\|^{2} \geq c\left\langle\Delta_{b} f, f\right\rangle
$$

for $f \in C^{2}\left(\mathscr{H}, \mathscr{V}_{1}\right) \cap L^{2}\left(\mathscr{H}, \mathscr{V}_{1}\right)$, where $\Delta_{b}$ is the SubLaplacian on the right quaternionic Heisenberg group. But $\left\langle\Delta_{b} f, f\right\rangle$ does not control the $L^{2}$ norm of $f$. It only controls $\|f\|^{2}{ }_{L} \frac{Q+2}{Q-2}$ by the well-known Sobolev inequality [19], where $Q=4 n+6$ is the homogeneous dimension of $\mathscr{H}$. To avoid this difficulty, we consider the locally flat compact manifold $\mathscr{H} / \mathscr{H}_{\mathbb{Z}}$, where

$$
\mathscr{H}_{\mathbb{Z}}:=\mathbb{Z}^{4 n+3}
$$

is a lattice of $\mathscr{H}$. It is a spherical qc manifold (cf. [31]). Because the self-adjoint subelliptic operator $\Delta_{b}$ over a compact manifold has discrete spectra, $\left\langle\Delta_{b} f, f\right\rangle$ controls the $L^{2}$ norm of $f$ for $f \perp$ ker $\Delta_{b}$. Moreover, by the Poincaré-type inequality we can show ker $\Delta_{b}$ consisting of constant vectors. Namely, there exists some $c^{\prime \prime}>0$ such that

$$
\left\langle\Delta_{b} f, f\right\rangle \geq c^{\prime \prime}\|f\|^{2}
$$

for $f \in C^{2}\left(\mathscr{H} / \mathscr{H}_{\mathbb{Z}}, \mathscr{V}_{1}\right)$ and $f \perp$ constant vectors. It is a standard way to use the $L^{2}$ estimate to solve the nonhomogeneous tangential $k$-Cauchy-Fueter equation (1.7)-(1.8) on $\mathscr{H} / \mathscr{H}_{\mathbb{Z}}$. The solution has an important vanishing property which allows us to prove Hartogs' phenomenon. See also [13] for the existence theorem for $\bar{\partial}_{b}$-equation over compact CR manifolds by establishing a priori estimate.

In Sect. 2, we give preliminaries on the right quaternionic Heisenberg group, the horizontal complex vector fields $Z_{A}^{A^{\prime}}$ 's and nice behavior of their commutators. We also give the definition of the tangential $k$-Cauchy-Fueter operators and their basic properties. It is checked directly that (1.4)-(1.5) is a complex. We compare the complexes on the left and right quaternionic Heisenberg groups. In Sect. 3, we use integration by part and Poincaré-type inequality to show the $L^{2}$ estimate (1.10) (1.12) for the tangential $k$-Cauchy-Fueter operator. In Sect. 4 , we use the $L^{2}$ estimate to solve the nonhomogeneous tangential $k$-Cauchy-Fueter equation (1.7)-(1.8) over the quotient manifold $\mathscr{H} / \mathscr{H}_{\mathbb{Z}}$ and derive the Hartogs' phenomenon. In Sect. 5, we construct the nilpotent Lie groups of step two associated with quadratic hypersurfaces. By constructing a diffeomorphism from the group $\mathscr{H}$ to the hypersurface $\mathcal{S}$ in (1.1), we show that the pushforward of the tangential $k$-Cauchy-Fueter operator on the group $\mathscr{H}$ coincides with the restriction of the $k$-Cauchy-Fueter operator on $\mathbb{H}^{n+1}$ to this hypersurface. Therefore, the restriction of a $k$-regular functions to $\mathcal{S}$ is $k$-CF on $\mathscr{H}$. $k$-CF functions are abundant because so are $k$-regular functions on $\mathbb{H}^{n+1}$ [21]. In Appendix, we give the expression of $\square_{1}$ for $n=2, k=2$. 


\section{The tangential $k$-Cauchy-Fueter complexes}

\subsection{The right quaternionic Heisenberg group $\mathscr{H}$ and the locally flat compact manifold $\mathscr{H} / \mathscr{H}_{\mathbb{Z}}$}

The multiplication of the right quaternionic Heisenberg group $\mathscr{H}$ can be written in terms of real variables (cf. [36, (2.13)]) as

$$
(x, t) \cdot(y, s)=\left(x+y, t_{\beta}+s_{\beta}+2 \sum_{l=0}^{n-1} \sum_{j, k=1}^{4} B_{k j}^{\beta} x_{4 l+k} y_{4 l+j}\right),
$$

for $x, y \in \mathbb{R}^{4 n}, t, s \in \mathbb{R}^{3}, \beta=1,2,3$, where $B_{k j}^{\beta}$ is the $(k, j)$ th entry of the following matrices

$$
\begin{aligned}
B^{1}: & =\left(\begin{array}{cccc}
0 & -1 & 0 & 0 \\
1 & 0 & 0 & 0 \\
0 & 0 & 0 & -1 \\
0 & 0 & 1 & 0
\end{array}\right), B^{2}:=\left(\begin{array}{cccc}
0 & 0 & -1 & 0 \\
0 & 0 & 0 & 1 \\
1 & 0 & 0 & 0 \\
0 & -1 & 0 & 0
\end{array}\right), \\
B^{3} & :=\left(\begin{array}{cccc}
0 & 0 & 0 & -1 \\
0 & 0 & -1 & 0 \\
0 & 1 & 0 & 0 \\
1 & 0 & 0 & 0
\end{array}\right),
\end{aligned}
$$

satisfying the commutating relation of quaternions $\left(B^{1}\right)^{2}=\left(B^{2}\right)^{2}=\left(B^{3}\right)^{2}=$ $-i d, B^{1} B^{2}=B^{3}$. This is because for $x=x_{1}+x_{2} \mathbf{i}+x_{3} \mathbf{j}+x_{4} \mathbf{k}$ and $x^{\prime}=x_{1}^{\prime}+x_{2}^{\prime} \mathbf{i}+x_{3}^{\prime} \mathbf{j}+x_{4}^{\prime} \mathbf{k}$, we have

$$
\begin{aligned}
\operatorname{Im}\left(x \overline{x^{\prime}}\right)= & \left(-x_{1} x_{2}^{\prime}+x_{2} x_{1}^{\prime}-x_{3} x_{4}^{\prime}+x_{4} x_{3}^{\prime}\right) \mathbf{i}+\left(-x_{1} x_{3}^{\prime}+x_{3} x_{1}^{\prime}+x_{2} x_{4}^{\prime}-x_{4} x_{2}^{\prime}\right) \mathbf{j} \\
& +\left(-x_{1} x_{4}^{\prime}+x_{4} x_{1}^{\prime}-x_{2} x_{3}^{\prime}+x_{3} x_{2}^{\prime}\right) \mathbf{k}=\sum_{\beta=1}^{3} \sum_{k, j=1}^{4} B_{k j}^{\beta} x_{k} x_{j}^{\prime} \mathbf{i}_{\beta},
\end{aligned}
$$

where $\mathbf{i}_{0}=1, \mathbf{i}_{1}=\mathbf{i}, \mathbf{i}_{2}=\mathbf{j}, \mathbf{i}_{3}=\mathbf{k}$. For fixed point $(y, s) \in \mathscr{H}$, the left translate $\tau_{(y, s)}: \mathscr{H} \longrightarrow \mathscr{H},(x, t) \longmapsto(y, s) \cdot(x, t)$, is an affine transformation given by a lower triangular matrix by (2.1). So the Lebesgue measure on $\mathbb{R}^{4 n+3}$ is an invariant measure under the left translation of $\mathscr{H}$. Recall that we have the following left invariant vector fields on $\mathscr{H}$ :

$$
\left(Y_{a} f\right)(y, s)=\left.\frac{\mathrm{d}}{\mathrm{d} t} f\left((y, s)\left(t e_{a}, 0\right)\right)\right|_{t=0}, \quad a=1,2, \ldots, 4 n,
$$

where $e_{a}$ is $(0, \ldots, 1, \ldots, 0)$ with only the $a$ th entry equal to 1 . Then,

$$
Y_{4 l+j}:=\frac{\partial}{\partial y_{4 l+j}}+2 \sum_{\beta=1}^{3} \sum_{k=1}^{4} B_{k j}^{\beta} y_{4 l+k} \frac{\partial}{\partial s_{\beta}},
$$

whose brackets are

$$
\left[Y_{4 l+k}, Y_{4 l+j}\right]=4 \sum_{\beta=1}^{3} B_{k j}^{\beta} \partial_{s_{\beta}}, \quad \text { and }\left[Y_{4 l+k}, Y_{4 l^{\prime}+j}\right]=0 \text { for } l \neq l^{\prime},
$$


where $l, l^{\prime}=0,1, \ldots, n-1, j, k=1, \ldots, 4$. The SubLaplacian is defined as

$$
\Delta_{b}:=-\sum_{a=1}^{4 n} Y_{a}^{2}
$$

The norm of the right quaternionic Heisenberg group $\mathscr{H}$ is defined by

$$
\|(y, s)\|:=\left(|y|^{4}+|s|^{2}\right)^{\frac{1}{4}} .
$$

Define balls $B(\xi, r):=\left\{\eta \in \mathscr{H} ;\left\|\xi^{-1} \cdot \eta\right\|<r\right\}$ for $\xi \in \mathscr{H}, r>0$. The fundamental set of $\mathscr{H}$ under the action of the lattice $\mathscr{H}_{\mathbb{Z}}$ in (1.11) is

$$
\mathscr{F}=\left\{(y, s) \in \mathscr{H} \mid 0 \leq y_{a}<1,0 \leq s_{\beta}<1, a=1, \ldots, 4 n, \beta=1,2,3\right\} .
$$

$\mathscr{H} / \mathscr{H}_{\mathbb{Z}}$ is equivalent to $\mathscr{F}$ as a set.

Proposition $2.1 \mathscr{H}$ is the disjoint union of $\tau_{(n, m)} \mathscr{F}$ with $(n, m) \in \mathscr{H}_{\mathbb{Z}}$.

Proof We need to prove that for any $(y, s) \in \mathscr{H}$, there exist unique $\left(y^{\prime}, s^{\prime}\right) \in \mathscr{F}$ and $(n, m) \in \mathscr{H}_{\mathbb{Z}}$ such that $(y, s)=(n, m) \cdot\left(y^{\prime}, s^{\prime}\right)$. Let $\left(n_{a}, m_{a}\right) \in \mathscr{H}_{\mathbb{Z}}, a=1,2$. By the multiplication law (2.1), we have

$$
\left(n_{a}, m_{a}\right) \cdot(y, s)=\left(n_{a}+y,\left(m_{a}\right)_{\beta}+s_{\beta}+2 \sum_{l=0}^{n-1} \sum_{j, k=1}^{4} B_{k j}^{\beta}\left(n_{a}\right)_{4 l+k} y_{4 l+j}\right) .
$$

If $n_{1} \neq n_{2}$, the $y$-coordinates of $\left(n_{1}, m_{1}\right) \cdot(y, s)$ and $\left(n_{2}, m_{2}\right) \cdot(y, s)$ are $n_{1}+y$ and $n_{2}+y$, respectively, which are different. If $n_{1}=n_{2}, m_{1} \neq m_{2}$, we see that their $s$-coordinates in (2.9) must be different. This proves the uniqueness.

For $(y, s)=\left(y_{1}, \ldots, y_{4 n}, s_{1}, s_{2}, s_{3}\right)$, we can choose $y^{\prime} \in \mathbb{R}^{4 n}$ with $0 \leq y_{j}^{\prime}<1$ and $n \in \mathbb{Z}^{4 n}$ such that $y_{j}=n_{j}+y_{j}^{\prime}$. Then, we can determine $s^{\prime} \in \mathbb{R}^{3}$ and $m \in \mathbb{Z}^{3}$ satisfying

$$
m_{\beta}+s_{\beta}^{\prime}=s_{\beta}-2 \sum_{l=0}^{n-1} \sum_{j, k=1}^{4 n} B_{k j}^{\beta} n_{4 l+k} y_{4 l+j}^{\prime}, \quad \text { with } 0 \leq s_{\beta}^{\prime}<1,
$$

for $\beta=1,2,3$. So $\mathscr{H}$ is the disjoint union of $\tau_{(n, m)} \mathscr{F}$. The proposition is proved.

$\mathscr{H} / \mathscr{H}_{\mathbb{Z}}$ has the structure of a locally flat manifold as follows (cf. [22, p. 238]). Let $\pi: \mathscr{H} \rightarrow$ $\mathscr{H} / \mathscr{H}_{\mathbb{Z}}$ be the projection. We can find a finite number of balls $B\left(\xi_{j}, r\right), j=1, \ldots, N$, covering $\mathscr{F}$ with $r$ sufficiently small so that $\tau_{(n, m)} B\left(\xi_{j}, r\right) \cap B\left(\xi_{j}, r\right)=\emptyset$ for any $(0,0) \neq$ $(n, m) \in \mathscr{H}_{\mathbb{Z}}$. Note that $\pi B\left(\xi_{i}, r\right) \cap \pi B\left(\xi_{j}, r\right) \neq \emptyset$ for $i \neq j$ if and only if there exist unique $(n, m) \in \mathscr{H}_{\mathbb{Z}}$, such that

$$
\tau_{(n, m)} B\left(\xi_{i}, r\right) \cap B\left(\xi_{j}, r\right) \neq \emptyset .
$$

Then, we can construct coordinates charts $\left(\pi B\left(\xi_{j}, r\right), \phi_{j}\right)$, where $\phi_{j}: \pi B\left(\xi_{j}, r\right) \rightarrow$ $B\left(\xi_{j}, r\right)$ and the transition function $\phi_{j} \circ \phi_{i}^{-1}$ is given by $\tau_{(n, m)}$ for some $(n, m) \in \mathscr{H}_{\mathbb{Z}}$ such that (2.10) holds.

A function is called periodic on $\mathscr{H}$ if

$$
f(y, s)=f((n, m)(y, s))
$$


for any $(n, m) \in \mathscr{H}_{\mathbb{Z}}$. A function over $\mathscr{H} / \mathscr{H}_{\mathbb{Z}}$ can be viewed as a function on $\mathscr{F}$ and be extended to a periodic function on $\mathscr{H}$ by

$$
f(y, s)=f\left((n, m) \cdot\left(y^{\prime}, s^{\prime}\right)\right)=f\left(y^{\prime}, s^{\prime}\right),
$$

for $(y, s)=(n, m) \cdot\left(y^{\prime}, s^{\prime}\right)$ and $\left(y^{\prime}, s^{\prime}\right) \in \mathscr{F}$. If $f$ is periodic, then so is $Y_{a} f$ for any $a$. This is because

$$
\begin{aligned}
\left(Y_{a} f\right)\left(y^{\prime}, s^{\prime}\right) & =\left.\frac{\mathrm{d}}{\mathrm{d} t} f\left(\left(y^{\prime}, s^{\prime}\right)\left(t e_{a}, 0\right)\right)\right|_{t=0}=\left.\frac{\mathrm{d}}{\mathrm{d} t} f\left((n, m)\left(y^{\prime}, s^{\prime}\right)\left(t e_{a}, 0\right)\right)\right|_{t=0} \\
& =\left(Y_{a} f\right)(y, s),
\end{aligned}
$$

for $e_{a}$ as in (2.3). Thus, the action of $Y_{a}$ on functions over $\mathscr{H} / \mathscr{H}_{\mathbb{Z}}$ is well-defined, i.e., it is a vector field over $\mathscr{H} / \mathscr{H}_{\mathbb{Z}}$.

\subsection{Complex horizontal vector fields $Z_{A}^{A^{\prime}}$ 's and the tangential $k$-Cauchy-Fueter operator}

We consider the following complex horizontal left invariant vector fields on $\mathscr{H}$ :

$$
\left(Z_{A A^{\prime}}\right):=\left(\begin{array}{cc}
Y_{1}+\mathbf{i} Y_{2} & -Y_{3}-\mathbf{i} Y_{4} \\
Y_{3}-\mathbf{i} Y_{4} & Y_{1}-\mathbf{i} Y_{2} \\
\vdots & \vdots \\
Y_{4 l+1}+\mathbf{i} Y_{4 l+2} & -Y_{4 l+3}-\mathbf{i} Y_{4 l+4} \\
Y_{4 l+3}-\mathbf{i} Y_{4 l+4} & Y_{4 l+1}-\mathbf{i} Y_{4 l+2} \\
\vdots & \vdots
\end{array}\right)
$$

where $A=0,1, \ldots, 2 n-1, A^{\prime}=0^{\prime}, 1^{\prime}$. It is motivated by the embedding $\tau$ of quaternionic algebra $\mathbb{H}$ into $\mathfrak{g l}(2, \mathbb{C})$ :

$$
\tau\left(x_{1}+x_{2} \mathbf{i}+x_{3} \mathbf{j}+x_{4} \mathbf{k}\right)=\left(\begin{array}{rr}
x_{1}+\mathbf{i} x_{2} & -x_{3}-\mathbf{i} x_{4} \\
x_{3}-\mathbf{i} x_{4} & x_{1}-\mathbf{i} x_{2}
\end{array}\right)
$$

and vector fields

$$
\left(\nabla_{A A^{\prime}}\right):=\left(\begin{array}{cc}
\partial_{x_{1}}+\mathbf{i} \partial_{x_{2}} & -\partial_{x_{3}}-\mathbf{i} \partial_{x_{4}} \\
\partial_{x_{3}}-\mathbf{i} \partial_{x_{4}} & \partial_{x_{1}}-\mathbf{i} \partial_{x_{2}} \\
\vdots & \vdots \\
\partial_{x_{4 l+1}}+\mathbf{i} \partial_{x_{4 l+2}} & -\partial_{x_{4 l+3}}-\mathbf{i} \partial_{x_{4 l+4}} \\
\partial_{x_{4 l+3}}-\mathbf{i} \partial_{x_{4 l+4}} & \partial_{x_{4 l+1}}-\mathbf{i} \partial_{x_{4 l+2}} \\
\vdots & \vdots
\end{array}\right)
$$

to construct the $k$-Cauchy-Fueter operators on $\mathbb{H}^{n+1}$ in [35]. We will use matrices

$$
\left(\varepsilon_{A^{\prime} B^{\prime}}\right)=\left(\begin{array}{cc}
0 & 1 \\
-1 & 0
\end{array}\right), \quad\left(\varepsilon^{A^{\prime} B^{\prime}}\right)=\left(\begin{array}{cc}
0 & -1 \\
1 & 0
\end{array}\right)
$$

to raise or lower primed indices, e.g., $Z_{A}^{A^{\prime}}=\sum_{B^{\prime}=0^{\prime}, 1^{\prime}} Z_{A B^{\prime}} \varepsilon^{B^{\prime} A^{\prime}}$. Here, $\left(\varepsilon^{A^{\prime} B^{\prime}}\right)$ is the inverse of $\left(\varepsilon_{A^{\prime} B^{\prime}}\right)$. Then,

$$
Z_{A}^{0^{\prime}}=Z_{A 1^{\prime}}, \quad Z_{A}^{1^{\prime}}=-Z_{A 0^{\prime}},
$$


and

$$
\left(Z_{A}^{A^{\prime}}\right)=\left(\begin{array}{cc}
\vdots & \vdots \\
Z_{2 l}^{0^{\prime}} & Z_{2 l}^{1^{\prime}} \\
Z_{2 l+1}^{0^{\prime}} & Z_{2 l+1}^{1^{\prime}} \\
\vdots & \vdots
\end{array}\right)=\left(\begin{array}{cc}
\vdots & \vdots \\
-Y_{4 l+3}-\mathbf{i} Y_{4 l+4} & -Y_{4 l+1}-\mathbf{i} Y_{4 l+2} \\
Y_{4 l+1}-\mathbf{i} Y_{4 l+2} & -Y_{4 l+3}+\mathbf{i} Y_{4 l+4} \\
\vdots & \vdots
\end{array}\right) .
$$

An element of $\mathbb{C}^{2}$ is denoted by $\left(f_{A^{\prime}}\right)$ with $A^{\prime}=0^{\prime}, 1^{\prime}$. The symmetric power $\odot{ }^{p} \mathbb{C}^{2}$ is a subspace of $\otimes^{p} \mathbb{C}^{2}$, whose element is a $2^{p}$-tuple $\left(f_{A_{1}^{\prime} A_{2}^{\prime} \ldots A_{p}^{\prime}}\right)$ with $A_{1}^{\prime}, A_{2}^{\prime}, \ldots, A_{p}^{\prime}=0^{\prime}, 1^{\prime}$, such that $f_{A_{1}^{\prime} A_{2}^{\prime} \ldots A_{p}^{\prime}} \in \mathbb{C}$ are invariant under permutations of subscripts, i.e.,

$$
f_{A_{1}^{\prime} A_{2}^{\prime} \ldots A_{p}^{\prime}}=f_{A_{\sigma(1)}^{\prime}} A_{\sigma(2)}^{\prime} \ldots A_{\sigma(p)}^{\prime}
$$

for any $\sigma$ in the group $S_{p}$ of permutations of $p$ letters. An element of $\odot{ }^{p} \mathbb{C}^{2} \otimes \wedge^{q} \mathbb{C}^{2 n}$ is given by a tuple $\left(f_{A_{1}^{\prime} \ldots A_{p}^{\prime} A_{1} \ldots A_{q}}\right) \in\left(\otimes^{p} \mathbb{C}^{2}\right) \otimes\left(\otimes^{q} \mathbb{C}^{2 n}\right)$, which is invariant under permutations of subscripts of $A_{1}^{\prime}, \ldots, A_{p}^{\prime}$, and antisymmetric under permutations of subscripts of $A_{1}, \ldots, A_{q}=0,1, \ldots 2 n-1$. In the sequel, we will write $f_{A A_{2}^{\prime} A_{3}^{\prime} \ldots A_{k}^{\prime}}:=f_{A_{2}^{\prime} A_{3}^{\prime} \ldots A_{k}^{\prime} A}$ and $f_{A_{3}^{\prime} \ldots A_{k}^{\prime} A B}:=f_{A B A_{3}^{\prime} \ldots A_{k}^{\prime}}$ for convenience. We will use symmetrization of primed indices

$$
f_{\ldots\left(A_{1}^{\prime} \ldots A_{p}^{\prime}\right) \ldots}:=\frac{1}{p !} \sum_{\sigma \in S_{p}} f_{\ldots A_{\sigma(1)}^{\prime} \ldots A_{\sigma(p)}^{\prime} \ldots} .
$$

The tangential $k$-Cauchy-Fueter operator in (1.4) is given by

$$
\left(\mathscr{D}_{0} f\right)_{A A_{2}^{\prime} \ldots A_{k}^{\prime}}:=\sum_{A_{1}^{\prime}=0^{\prime}, 1^{\prime}} Z_{A}^{A_{1}^{\prime}} f_{A_{1}^{\prime} A_{2}^{\prime} \ldots A_{k}^{\prime}},
$$

for $f \in C^{1}\left(\Omega, \mathscr{V}_{0}\right)$. The $k$-Cauchy-Fueter operator on $\mathbb{H}^{n+1}[35]$ is $\widehat{\mathscr{D}}_{0}: C^{1}\left(\mathbb{H}^{n+1}, \mathscr{V}_{0}\right) \rightarrow$ $C^{1}\left(\mathbb{H}^{n+1}, \mathscr{V}_{1}\right)$ with

$$
\left(\widehat{\mathscr{D}}_{0} f\right)_{A_{2}^{\prime} \ldots A_{k}^{\prime} A}:=\sum_{B^{\prime}=0^{\prime}, 1^{\prime}} \nabla_{A}^{B^{\prime}} f_{B^{\prime} A_{2}^{\prime} \ldots A_{k}^{\prime}},
$$

where $\nabla$ is given by (2.14). A $\mathscr{V}_{0}$-valued distribution $f$ is called $k$-regular on $\Omega \in \mathbb{H}^{n+1}$ if $\widehat{\mathscr{D}}_{0} f=0$ on $\Omega$ in the sense of distributions.

\subsection{Commutators of complex horizontal vector fields}

The following nice behavior of commutators of $Z_{A}^{A^{\prime}}$ 's plays a very important role to show that (1.4) is a complex and to establish the $L^{2}$ estimate. It is also the reason why the tangential $k$-Cauchy-Fueter complex on the right Heisenberg group is simpler than that on the left one.

Lemma 2.1 (1) Vector fields in each column in (2.16) are commutative, i.e., for fixed $A^{\prime}=$ $0^{\prime}$ or $1^{\prime}$,

$$
\left[Z_{A}^{A^{\prime}}, Z_{B}^{A^{\prime}}\right]=0,
$$

for any $A, B=0, \ldots, 2 n-1$. 
(2) We have

$$
\begin{aligned}
& {\left[Z_{2 l}^{0^{\prime}}, Z_{2 l}^{1^{\prime}}\right]=\overline{\left[Z_{2 l+1}^{0^{\prime}}, Z_{2 l+1}^{1^{\prime}}\right]}=8\left(\partial_{s_{2}}+\mathbf{i} \partial_{s_{3}}\right),} \\
& {\left[Z_{2 l}^{0^{\prime}}, Z_{2 l+1}^{1^{\prime}}\right]=\left[Z_{2 l+1}^{0^{\prime}}, Z_{2 l}^{1^{\prime}}\right]=8 \mathbf{i} \partial_{s_{1}},}
\end{aligned}
$$

$l=0, \ldots, n-1$, and any other bracket vanishes.

Proof (1) If $\{A, B\} \neq\{2 l, 2 l+1\}$ for any integer $l$, we have

$$
\left[Z_{A}^{A^{\prime}}, Z_{B}^{B^{\prime}}\right]=0, \text { for } A^{\prime}, B^{\prime}=0^{\prime}, 1^{\prime},
$$

by using (2.5) because $Z_{A}^{A^{\prime}}$ and $Z_{B}^{B^{\prime}}$ only involve $Y_{4 l+j}$ 's for different $l$. It follows from (2.2) (2.5) that

$$
\begin{aligned}
& {\left[Y_{4 l+1}, Y_{4 l+2}\right]=\left[Y_{4 l+3}, Y_{4 l+4}\right]=-4 \partial_{s_{1}},} \\
& {\left[Y_{4 l+1}, Y_{4 l+3}\right]=-\left[Y_{4 l+2}, Y_{4 l+4}\right]=-4 \partial_{s_{2}},} \\
& {\left[Y_{4 l+1}, Y_{4 l+4}\right]=\left[Y_{4 l+2}, Y_{4 l+3}\right]=-4 \partial_{s_{3}} .}
\end{aligned}
$$

Then, for $\{A, B\}=\{2 l, 2 l+1\}$, we have

$$
\begin{aligned}
{\left[Z_{2 l}^{0^{\prime}}, Z_{2 l+1}^{0^{\prime}}\right] } & =\left[-Y_{4 l+3}-\mathbf{i} Y_{4 l+4}, Y_{4 l+1}-\mathbf{i} Y_{4 l+2}\right] \\
& =\left[Y_{4 l+1}, Y_{4 l+3}\right]+\left[Y_{4 l+2}, Y_{4 l+4}\right]-\mathbf{i}\left[Y_{4 l+2}, Y_{4 l+3}\right]+\mathbf{i}\left[Y_{4 l+1}, Y_{4 l+4}\right]=0, \\
{\left[Z_{2 l}^{1^{\prime}}, Z_{2 l+1}^{1^{\prime}}\right] } & =\left[-Y_{4 l+1}-\mathbf{i} Y_{4 l+2},-Y_{4 l+3}+\mathbf{i} Y_{4 l+4}\right] \\
& =\left[Y_{4 l+1}, Y_{4 l+3}\right]+\left[Y_{4 l+2}, Y_{4 l+4}\right]+\mathbf{i}\left[Y_{4 l+2}, Y_{4 l+3}\right]-\mathbf{i}\left[Y_{4 l+1}, Y_{4 l+4}\right]=0,
\end{aligned}
$$

by (2.21). Then, (2.19) follows.

(2) Similarly, we have

$$
\begin{aligned}
{\left[Z_{2 l}^{0^{\prime}}, Z_{2 l}^{1^{\prime}}\right]=} & {\left[-Y_{4 l+3}-\mathbf{i} Y_{4 l+4},-Y_{4 l+1}-\mathbf{i} Y_{4 l+2}\right] } \\
= & -\left[Y_{4 l+1}, Y_{4 l+3}\right]+\left[Y_{4 l+2}, Y_{4 l+4}\right] \\
& -\mathbf{i}\left[Y_{4 l+2}, Y_{4 l+3}\right]-\mathbf{i}\left[Y_{4 l+1}, Y_{4 l+4}\right]=8\left(\partial_{s_{2}}+\mathbf{i} \partial_{s_{3}}\right), \\
{\left[Z_{2 l+1}^{0^{\prime}}, Z_{2 l+1}^{1^{\prime}}\right]=} & \overline{\left[Z_{2 l}^{0^{\prime}}, Z_{2 l}^{1^{\prime}}\right]}=8\left(\partial_{s 2}-\mathbf{i} \partial_{s 3}\right), \\
{\left[Z_{2 l}^{0^{\prime}}, Z_{2 l+1}^{1^{\prime}}\right]=} & {\left[-Y_{4 l+3}-\mathbf{i} Y_{4 l+4},-Y_{4 l+3}+\mathbf{i} Y_{4 l+4}\right]=-2 \mathbf{i}\left[Y_{4 l+3}, Y_{4 l+4}\right]=8 \mathbf{i} \partial_{s_{1}}, } \\
{\left[Z_{2 l+1}^{0^{\prime}}, Z_{2 l}^{1^{\prime}}\right]=} & {\left[Y_{4 l+1}-\mathbf{i} Y_{4 l+2},-Y_{4 l+1}-\mathbf{i} Y_{4 l+2}\right]=-2 \mathbf{i}\left[Y_{4 l+1}, Y_{4 l+2}\right]=8 \mathbf{i} \partial_{s_{1}}, }
\end{aligned}
$$

by (2.21). The lemma is proved.

On the left quaternionic Heisenberg group, vector fields in each column are not commutative (2.43)-(2.44). We have the following corollary directly by Lemma 2.1 (2).

\section{Corollary 2.1}

$$
\left[Z_{A}^{0^{\prime}}, Z_{B}^{1^{\prime}}\right]+\left[Z_{A}^{1^{\prime}}, Z_{B}^{0^{\prime}}\right]=0
$$

for any $A, B=0, \ldots, 2 n-1$. 


\subsection{The tangential $k$-Cauchy-Fueter complex}

Differential operators in the complex (1.4) are as follows. For $j=0,1, \ldots, k-1, \mathscr{D}_{j}$ : $C^{\infty}\left(\Omega, \mathscr{V}_{j}\right) \rightarrow C^{\infty}\left(\Omega, \mathscr{V}_{j+1}\right)$ with $\mathscr{V}_{j}=\odot^{k-j} \mathbb{C}^{2} \otimes \wedge^{j} \mathbb{C}^{2 n}$ is a differential operator of the first order given by

$$
\left(\mathscr{D}_{j} f\right)_{A_{0} \ldots A_{j} A_{1}^{\prime} \ldots A_{k-j-1}^{\prime}}=(j+1) \sum_{A^{\prime}=0^{\prime}, 1^{\prime}} Z_{\left[A_{0}\right.}^{A^{\prime}} f_{\left.A_{1} \ldots A_{j}\right] A^{\prime} A_{1}^{\prime} \ldots A_{k-j-1}^{\prime}},
$$

where $\left[A_{0} A_{1} \ldots A_{j}\right]$ is the antisymmetrization of unprimed indices given by

$$
f_{\ldots\left[A_{1} \ldots A_{p}\right] \ldots}:=\frac{1}{p !} \sum_{\sigma \in S_{p}} \operatorname{sign}(\sigma) f_{\ldots A_{\sigma(1)} \ldots A_{\sigma(p)} \ldots} .
$$

In particular, $h_{[A B]}:=\frac{1}{2}\left(h_{A B}-h_{B A}\right)$. By definition, we have

$$
f_{\ldots[}\left[A_{1} \ldots\left[A_{j} \ldots A_{l}\right] \ldots A_{p}\right] \ldots=f_{\ldots\left[A_{1} \ldots A_{j} \ldots A_{l} \ldots A_{p}\right] \ldots .} .
$$

$\mathscr{D}_{k}: C^{\infty}\left(\Omega, \mathscr{V}_{k}\right) \rightarrow C^{\infty}\left(\Omega, \mathscr{V}_{k+1}\right)$ with $\mathscr{V}_{k}=\wedge^{k} \mathbb{C}^{2 n}$ and $\mathscr{V}_{k+1}=\wedge^{k+2} \mathbb{C}^{2 n}$ is a differential operator of the second order given by

$$
\left(\mathscr{D}_{k} f\right)_{A_{1} \ldots A_{k+2}}=(k+2) Z_{\left[A_{1}\right.}^{0^{\prime}} Z_{A_{2}}^{1^{\prime}} f_{\left.A_{3} \ldots A_{k+2}\right]} .
$$

For $j=k+1, \ldots, 2 n-2, \mathscr{D}_{j}: C^{\infty}\left(\Omega, \mathscr{V}_{j}\right) \rightarrow C^{\infty}\left(\Omega, \mathscr{V}_{j+1}\right)$ with $\mathscr{V}_{j}=\odot^{j-k-1} \mathbb{C}^{2} \otimes$ $\wedge^{j+1} \mathbb{C}^{2 n}$ is a differential operator of the first order given by

$$
\left(\mathscr{D}_{j} f\right)_{A_{1} \ldots A_{j+2}}^{A_{1}^{\prime} \ldots A_{j-k}^{\prime}}=(j+2) Z_{\left[A_{1}\right.}^{\left(A_{1}^{\prime}\right.} f_{\left.A_{2} \ldots A_{j+2}\right]}^{\left.A_{2}^{\prime} \ldots A_{j-k}^{\prime}\right)} .
$$

Remark 2.1 The $k$-Cauchy-Fueter complex on $\mathbb{H}^{n}[35,41]$ is the same as (1.4)-(1.5) with $\mathscr{H}$ replaced by $\mathbb{H}^{n}$ and $Z_{A}^{A^{\prime}}$ in definition of $\mathscr{D}_{j}$ 's in (2.23), (2.26) and (2.27) replaced by $\nabla_{A}^{A^{\prime}}$ in (2.14).

\section{Lemma 2.2}

$$
Z_{[A}^{\left(A^{\prime}\right.} Z_{B]}^{\left.B^{\prime}\right)}=0
$$

for any $A, B=0, \ldots, 2 n-1$ and $A^{\prime}, B^{\prime}=0^{\prime}, 1^{\prime}$.

Proof Note that

$$
2 Z_{[A}^{A^{\prime}} Z_{B]}^{A^{\prime}}=Z_{A}^{A^{\prime}} Z_{B}^{A^{\prime}}-Z_{B}^{A^{\prime}} Z_{A}^{A^{\prime}}=\left[Z_{A}^{A^{\prime}}, Z_{B}^{A^{\prime}}\right]=0
$$

by (2.19), and

$$
\begin{aligned}
4 Z_{[A}^{\left(0^{\prime}\right.} Z_{B]}^{\left.1^{\prime}\right)} & =2 Z_{[A}^{0^{\prime}} Z_{B]}^{1^{\prime}}+2 Z_{[A}^{1^{\prime}} Z_{B]}^{0^{\prime}}=Z_{A}^{0^{\prime}} Z_{B}^{1^{\prime}}-Z_{B}^{0^{\prime}} Z_{A}^{1^{\prime}}+Z_{A}^{1^{\prime}} Z_{B}^{0^{\prime}}-Z_{B}^{1^{\prime}} Z_{A}^{0^{\prime}} \\
& =\left[Z_{A}^{0^{\prime}}, Z_{B}^{1^{\prime}}\right]+\left[Z_{A}^{1^{\prime}}, Z_{B}^{0^{\prime}}\right]=0,
\end{aligned}
$$

by Corollary 2.1. The lemma is proved.

Now, let us check (1.4) to be a complex by direct calculation as in [41, Section 3.1].

Theorem 2.1 (1.4) is a complex, i.e.,

$$
\mathscr{D}_{j+1} \circ \mathscr{D}_{j}=0
$$

for each $j$.

黛 Springer 
Proof For $A, B=0, \ldots, 2 n-1$ and $A_{3}^{\prime}, \ldots, A_{k}^{\prime}=0^{\prime}, 1^{\prime}$, we have

$$
\begin{aligned}
\left(\mathscr{D}_{1} \circ \mathscr{D}_{0} f\right)_{A B A_{3}^{\prime} \ldots A_{k}^{\prime}} & =2 \sum_{A^{\prime}=0^{\prime}, 1^{\prime}} Z_{[A}^{A^{\prime}}\left(\mathscr{D}_{0} f\right)_{B] A^{\prime} A_{3}^{\prime} \ldots A_{k}^{\prime}}=2 \sum_{A^{\prime}, C^{\prime}=0^{\prime}, 1^{\prime}} Z_{[A}^{A^{\prime}} Z_{B]}^{C^{\prime}} f_{C^{\prime} A^{\prime} A_{3}^{\prime} \ldots A_{k}^{\prime}} \\
& =2 \sum_{A^{\prime}, C^{\prime}=0^{\prime}, 1^{\prime}} Z_{[A}^{\left(A^{\prime}\right.} Z_{B]}^{\left.C^{\prime}\right)} f_{C^{\prime} A^{\prime} A_{3}^{\prime} \ldots A_{k}^{\prime}}=0,
\end{aligned}
$$

by Lemma 2.2 and $f_{C^{\prime} A^{\prime} A_{3}^{\prime} \ldots A_{k}^{\prime}}=f_{A^{\prime} C^{\prime} A_{3}^{\prime} \ldots A_{k}^{\prime}}$. For general $j=1, \ldots, k-2$, we have

$$
\begin{aligned}
& \left(\mathscr{D}_{j+1} \circ \mathscr{D}_{j} f\right)_{A_{1} \ldots A_{j+2}} A_{1}^{\prime} \ldots A_{k-j-2}^{\prime} \\
& =(j+2)(j+1) \sum_{A^{\prime}, C^{\prime}=0^{\prime}, 1^{\prime}} Z_{\left[A_{1}\right.}^{A^{\prime}} Z_{\left[A_{2}\right.}^{C^{\prime}} f_{\left.\left.A_{3} \ldots A_{j+2}\right]\right] C^{\prime} A^{\prime} A_{1}^{\prime} \ldots A_{k-j-2}^{\prime}} \\
& =(j+2)(j+1) \sum_{A^{\prime}, C^{\prime}=0^{\prime}, 1^{\prime}} Z_{\left[\left[A_{1}\right.\right.}^{\left(A^{\prime}\right.} Z_{\left.A_{2}\right]}^{\left.C^{\prime}\right)} f_{\left.A_{3} \ldots A_{j+2}\right] C^{\prime} A^{\prime} A_{1}^{\prime} \ldots A_{k-j-2}^{\prime}}=0,
\end{aligned}
$$

by using (2.25) repeatedly, Lemma 2.2 and $f$ symmetric in the primed indices again.

For $j=k-1$, we have

$$
\left(\mathscr{D}_{k} \circ \mathscr{D}_{k-1} f\right)_{A_{1} \ldots A_{k+2}}=(k+2) k \sum_{A^{\prime}=0^{\prime}, 1^{\prime}} Z_{\left[A_{1}\right.}^{0^{\prime}} Z_{A_{2}}^{1^{\prime}} Z_{\left[A_{3}\right.}^{A^{\prime}} f_{\left.\left.A_{4} \ldots A_{k+2}\right]\right] A^{\prime}}=0 .
$$

This is because if $A^{\prime}=1^{\prime}, Z_{\left[A_{1}\right.}^{0^{\prime}} Z_{\left[A_{2}\right.}^{1^{\prime}} Z_{\left.A_{3}\right]}^{1^{\prime}} f_{\left.A_{4} \ldots A_{k+2}\right] 1^{\prime}}=0$ by using (2.29), and if $A^{\prime}=0^{\prime}$,

$$
Z_{\left[A_{1}\right.}^{0^{\prime}} Z_{A_{2}}^{1^{\prime}} Z_{\left.A_{3}\right]}^{0^{\prime}}=Z_{\left[A_{1}\right.}^{0^{\prime}} Z_{\left[A_{2}\right.}^{1^{\prime}} Z_{\left.\left.A_{3}\right]\right]}^{0^{\prime}}=-Z_{\left[A_{1}\right.}^{0^{\prime}} Z_{\left[A_{2}\right.}^{0^{\prime}} Z_{\left.\left.A_{3}\right]\right]}^{1^{\prime}}=-Z_{\left[\left[A_{1}\right.\right.}^{0^{\prime}} Z_{\left.A_{2}\right]}^{0^{\prime}} Z_{\left.A_{3}\right]}^{1^{\prime}}=0,
$$

by using (2.25) repeatedly and Corollary 2.1 .

For $j=k$, we have

$$
\left(\mathscr{D}_{k+1} \circ \mathscr{D}_{k} f\right)_{A_{1} \ldots A_{k+3}}^{A^{\prime}}=(k+3)(k+2) Z_{\left[A_{1}\right.}^{A^{\prime}} Z_{\left[A_{2}\right.}^{0^{\prime}} Z_{A_{3}}^{1^{\prime}} f_{\left.\left.A_{4} \ldots A_{k+3}\right]\right]}=0 .
$$

This is because if $A^{\prime}=0^{\prime}, Z_{\left[\left[A_{1}\right.\right.}^{0^{\prime}} Z_{\left.A_{2}\right]}^{0^{\prime}} Z_{A_{3}}^{1^{\prime}} f_{\left.A_{4} \ldots A_{k+3}\right]}=0$ by using (2.29), and if $A^{\prime}=1^{\prime}$,

$$
Z_{\left[A_{1}\right.}^{1^{\prime}} Z_{A_{2}}^{0^{\prime}} Z_{\left.A_{3}\right]}^{1^{\prime}}=Z_{\left[A_{1}\right.}^{1^{\prime}} Z_{\left[A_{2}\right.}^{0^{\prime}} Z_{\left.\left.A_{3}\right]\right]}^{1^{\prime}{ }^{\prime}}=-Z_{\left[A_{1}\right.}^{1^{\prime}} Z_{\left[A_{2}\right.}^{1^{\prime}} Z_{\left.\left.A_{3}\right]\right]}^{0^{\prime}}=-Z_{\left[\left[A_{1}\right.\right.}^{1^{\prime}} Z_{\left.A_{2}\right]}^{1^{\prime}} Z_{\left.A_{3}\right]}^{0^{\prime}}=0,
$$

by using (2.25) repeatedly and Corollary 2.1 .

For $j=k+1, \ldots, 2 n-2$, we have

$$
\left(\mathscr{D}_{j+1} \circ \mathscr{D}_{j} f\right)_{A_{1} \ldots A_{j+3}}^{A_{1}^{\prime} \ldots A_{j-k+1}^{\prime}}=(j+3)(j+2) Z_{\left[\left[A_{1}\right.\right.}^{\left(\left(A_{1}^{\prime}\right.\right.} Z_{\left.A_{2}\right]}^{\left.A_{2}^{\prime}\right)} f_{\left.A_{3} \ldots A_{j+3}\right]}^{\left.A_{3}^{\prime} \ldots A_{j-k+1}^{\prime}\right)}=0,
$$

by Lemma 2.2. The theorem is proved.

\subsection{Comparison with the left case}

Recall that a transformation $T$ on $\mathscr{H}$ is called conformal if $\left\|T_{*} W_{1}\right\|=\left\|T_{*} W_{2}\right\|$ for any two horizontal vector fields $W_{1}$ and $W_{2}$ with $\left\|W_{1}\right\|=\left\|W_{2}\right\|$, where $\|W\|^{2}:=\sum_{j=1}^{4 n} a_{j}^{2}$ if we write $W=\sum_{j=1}^{4 n} a_{j} Y_{j}$. It is known that the group of conformal transformations on $\mathscr{H}$ is the real semisimple Lie group $\operatorname{Sp}(n+1,1)$ of rank one (cf., e.g., [18]) generated by the following transformations:

(1) Dilations:

$$
D_{\delta}:(y, s) \longrightarrow\left(\delta y, \delta^{2} s\right), \delta>0
$$


(2) Left translations:

$$
\tau_{(x, t)}:(y, s) \longrightarrow(x, t) \cdot(y, s)
$$

(3) Rotations:

$$
R_{\mathbf{a}}:(y, s) \longrightarrow(y \mathbf{a}, s), \text { for } \mathbf{a} \in \operatorname{Sp}(n),
$$

where

$$
\operatorname{Sp}(n)=\left\{\mathbf{a} \in \operatorname{GL}(n, \mathbb{H}) \mid \mathbf{a} \overline{\mathbf{a}}^{t}=I_{n}\right\}
$$

(4) The inversion:

$$
R:(y, s) \longrightarrow\left(-\left(|y|^{2}-s\right)^{-1} y, \frac{-s}{|y|^{4}+|s|^{2}}\right) ;
$$

(5) $\mathrm{Sp}(1)$ acts on $\mathscr{H}$ as

$$
\sigma:(y, s) \longrightarrow\left(\sigma y, \sigma s \sigma^{-1}\right),
$$

where the action on the first factor is left multiplication by $\sigma \in \mathbb{H}$ with $|\sigma|=1$, while the action on the second factor is isomorphism with $\mathrm{SO}(3)$.

It is known that $\operatorname{Sp}(n+1,1)$ is a real form of $\operatorname{Sp}(2(n+2), \mathbb{C})$, whose Lie algebra $\mathfrak{g}=\mathfrak{s p}(2(n+2), \mathbb{C})$ has the decomposition $\mathfrak{g}=\mathfrak{g}_{-2} \oplus \mathfrak{g}_{-1} \oplus \mathfrak{g}_{0} \oplus \mathfrak{g}_{1} \oplus \mathfrak{g}_{2}$, where $\mathfrak{g}_{-2}$ is an complex abelian subalgebra generated by $T_{1}, T_{2}, T_{3}$, and $\mathfrak{g}_{-1}$ is generated by $\left\{Y_{A A^{\prime}}\right\}, A=$ $0,1, \ldots, 2 n-1, A^{\prime}=0^{\prime}, 1^{\prime}$ with

$$
\begin{aligned}
& {\left[Y_{A 0^{\prime}}, Y_{(n+A) 0^{\prime}}\right]=4 T_{2},} \\
& {\left[Y_{A 1^{\prime}}, Y_{(n+A) 1^{\prime}}\right]=4 T_{3},} \\
& {\left[Y_{A 0^{\prime}}, Y_{(n+A) 1^{\prime}}\right]=\left[Y_{A 1^{\prime}}, Y_{(n+A) 0^{\prime}}\right]=4 T_{1},}
\end{aligned}
$$

and any other bracket vanishes (cf. [37, (2.10)]). $\mathfrak{p}:=\mathfrak{g}_{0} \oplus \mathfrak{g}_{1} \oplus \mathfrak{g}_{2}$ is a parabolic subgroup. $\mathfrak{u}_{-}:=\mathfrak{g}_{-2} \oplus \mathfrak{g}_{-1}$. Then,

$$
\mathfrak{g}=\mathfrak{u}_{-} \oplus \mathfrak{p} .
$$

Let $U_{-}$be the complex Lie group with Lie algebra $\mathfrak{u}_{-}$. There exist exact sequences [37, Theorem 3.2.1] on $\mathrm{U}_{-}$

$$
\begin{aligned}
0 & \rightarrow \mathcal{R}\left(\mathrm{U}_{-}, \odot^{k} \mathbb{C}^{2}\right) \stackrel{Q_{0}^{(k)}}{\longrightarrow} \mathcal{R}\left(\mathrm{U}_{-}, \odot^{k-1} \mathbb{C}^{2} \otimes V^{(1)}\right) \stackrel{Q_{1}^{(k)}}{\longrightarrow} \ldots \rightarrow \mathcal{R}\left(\mathrm{U}_{-}, V^{(k)}\right) \\
& \stackrel{Q_{k}^{(k)}}{\longrightarrow} \mathcal{R}\left(\mathrm{U}_{-}, V^{(k+2)}\right) \stackrel{Q_{k+1}^{(k)}}{\longrightarrow} \ldots \stackrel{Q_{2 n-1}^{(k)}}{\longrightarrow} \mathcal{R}\left(\mathrm{U}_{-}, \odot^{2 n-k} \mathbb{C}^{2}\right) \rightarrow 0,
\end{aligned}
$$

for $0 \leq k \leq n-2$, where operators $Q_{j}^{(k)}$, s are defined in terms of $Y_{A A^{\prime}}, T_{\beta}$ (cf. [37, Theorem 1.0.1]). Here, $V^{(j)}$ is the irreducible representation of $\mathfrak{s p}(2 n, \mathbb{C})$ with the highest weight to be the $j$ th fundamental weight $\omega_{j}$ and $\mathcal{R}\left(\mathrm{U}_{-}, V\right)$ is the space of $V$-valued polynomials over $\mathrm{U}_{-}$. These complexes are constructed by twistor method, and operators $Q_{j}^{(k)}$,s are invariant under $\operatorname{Sp}(2(\mathrm{n}+2), \mathbb{C})$.

The multiplication (1.6) of the left quaternionic Heisenberg group $\widetilde{\mathscr{H}}$ can be written as

$$
(x, t) \cdot(y, s)=\left(x+y, t_{\beta}+s_{\beta}+2 \sum_{l=0}^{n-1} \sum_{j, k=1}^{4} I_{k j}^{\beta} x_{4 l+k} y_{4 l+j}\right),
$$


for $x, y \in \mathbb{R}^{4 n}, t, s \in \mathbb{R}^{3}, \beta=1,2,3$, where $I_{k j}^{\beta}$ is the $(k, j)$ th entry of the following matrices

$$
\begin{aligned}
I^{1} & :=\left(\begin{array}{cccc}
0 & 1 & 0 & 0 \\
-1 & 0 & 0 & 0 \\
0 & 0 & 0 & -1 \\
0 & 0 & 1 & 0
\end{array}\right), I^{2}:=\left(\begin{array}{cccc}
0 & 0 & 1 & 0 \\
0 & 0 & 0 & 1 \\
-1 & 0 & 0 & 0 \\
0 & -1 & 0 & 0
\end{array}\right), \\
I^{3} & :=\left(\begin{array}{cccc}
0 & 0 & 0 & 1 \\
0 & 0 & -1 & 0 \\
0 & 1 & 0 & 0 \\
-1 & 0 & 0 & 0
\end{array}\right),
\end{aligned}
$$

satisfying the commutating relation of quaternions. Note that

$$
\tilde{X}_{4 l+j}=\frac{\partial}{\partial x_{4 l+j}}+2 \sum_{\beta=1}^{3} \sum_{k=1}^{4} I_{k j}^{\beta} x_{4 l+k} \frac{\partial}{\partial t_{\beta}}
$$

is standard left invariant vector field on $\widetilde{\mathscr{H}}$. Denote

$$
\left(\widetilde{Z}_{A A^{\prime}}\right):=\left(\begin{array}{cc}
\widetilde{X}_{1}+\mathbf{i} \widetilde{X}_{2} & -\widetilde{X}_{3}-\mathbf{i} \widetilde{X}_{4} \\
\widetilde{X}_{3}-\mathbf{i} \widetilde{X}_{4} & \widetilde{X}_{1}-\mathbf{i} \widetilde{X}_{2} \\
\vdots & \vdots \\
\widetilde{X}_{4 l+1}+\mathbf{i} \widetilde{X}_{4 l+2} & -\widetilde{X}_{4 l+3}-\mathbf{i} \widetilde{X}_{4 l+4} \\
\widetilde{X}_{4 l+3}-\mathbf{i} \widetilde{X}_{4 l+4} & \widetilde{X}_{4 l+1}-\mathbf{i} \widetilde{X}_{4 l+2} \\
\vdots & \vdots
\end{array}\right),
$$

where $A=0,1, \ldots, 2 n-1, A^{\prime}=0^{\prime}, 1^{\prime}$. They satisfy the following commutating relations:

$$
\begin{aligned}
& {\left[\widetilde{Z}_{(2 l) 0^{\prime}}, \widetilde{Z}_{(2 l+1) 0^{\prime}}\right]=8\left(\partial_{t_{2}}-\mathbf{i} \partial_{t_{3}}\right),} \\
& {\left[\widetilde{Z}_{(2 l) 1^{\prime}}, \widetilde{Z}_{(2 l+1) 1^{\prime}}\right]=8\left(\partial_{t_{2}}+\mathbf{i} \partial_{t_{3}}\right),} \\
& {\left[\widetilde{Z}_{(2 l) 0^{\prime}}, \widetilde{Z}_{(2 l+1) 1^{\prime}}\right]=\left[\widetilde{Z}_{(2 l) 1^{\prime}}, \widetilde{Z}_{(2 l+1) 0^{\prime}}\right]=-8 \mathbf{i} \partial_{t_{1}},}
\end{aligned}
$$

$l=0, \ldots, n-1$, and any other bracket vanishes. So by embedding the real Lie algebra of $\widetilde{\mathscr{H}}$ into the complex Lie algebra $\mathfrak{u}_{-}$by $\widetilde{Z}_{(2 l) A^{\prime}} \mapsto Y_{l A^{\prime}}, \widetilde{Z}_{(2 l+1) A^{\prime}} \mapsto Y_{(n+l) A^{\prime}}$ we get tangential $k$-Cauchy-Fueter complexes on $\widetilde{\mathscr{H}}$ (cf. [37, Theorem 1.0.1]), on which $G=\operatorname{Sp}(2(n+2), \mathbb{C})$ acts naturally.

Now, consider complexes on the right quaternionic Heisenberg group. We can show the following proposition as [38, Proposition 3.1].

Proposition 2.2 Under the transformation $M_{\mathbf{a}}: \mathbb{H}^{n} \rightarrow \mathbb{H}^{n}, q \mapsto q^{\prime}=q \mathbf{a}$ with $\mathbf{a}=\left(a_{j k}\right) \in$ $G L(n, \mathbb{H})$, where $q=\left(q_{1}, q_{2}, \ldots, q_{n}\right)$ with $q_{l+1}=x_{4 l+1}+\mathbf{i} x_{4 l+2}+\mathbf{j} x_{4 l+3}+\mathbf{k} x_{4 l+4}$, we have

$$
\bar{\partial}_{q_{l}}[f(q \mathbf{a})]=\sum_{m=1}^{n}\left[\bar{\partial}_{q_{m}^{\prime}}\left(\overline{\mathbf{a}}_{l m} f\right)\right](q \mathbf{a}),
$$

where $\bar{\partial}_{q_{l+1}}=\partial_{x_{4 l+1}}+\mathbf{i} \partial_{x_{4 l+2}}+\mathbf{j} \partial_{x_{4 l+3}}+\mathbf{k} \partial_{x_{4 l+4}}$.

Proof Denote $\widehat{q}=\left(x_{1}, \ldots, x_{4 n}\right)$. Since $M_{\mathrm{a}}$ defines a real linear transformation on the underlying vector space $\mathbb{R}^{4 n}$, we have $\widehat{q \mathbf{a}}=\widehat{q} \mathbf{a}^{\mathbb{R}}$ for some $(4 n) \times(4 n)$ real matrix $\mathbf{a}^{\mathbb{R}}$ associated with $\mathbf{a}$. As the $b$ th element of $\widehat{q \mathbf{a}}$ is $\sum_{a=1}^{4 n} x_{a} \mathbf{a}_{a b}^{\mathbb{R}}$, we have 


$$
\frac{\partial}{\partial x_{a}}[f(q \mathbf{a})]=\sum_{b=1}^{4 n} \frac{\partial f}{\partial x_{b}}(q \mathbf{a}) \mathbf{a}_{a b}^{\mathbb{R}} .
$$

Note that we can write $q_{l+1}=\sum_{j=1}^{4} \mathbf{i}_{j-1} x_{4 l+j}$. Therefore,

$$
\begin{aligned}
M_{\mathbf{a} *} \bar{\partial}_{q_{l+1}} & =\sum_{j=1}^{4} \mathbf{i}_{j-1} M_{\mathbf{a} *} \frac{\partial}{\partial x_{4 l+j}}=\sum_{b=1}^{4 n} \sum_{j=1}^{4} \mathbf{i}_{j-1} \frac{\partial}{\partial x_{b}} \mathbf{a}_{(4 l+j) b}^{\mathbb{R}} \\
& =\sum_{b=1}^{4 n} \sum_{j=1}^{4} \mathbf{i}_{j-1} \frac{\partial}{\partial x_{b}}\left(\mathbf{a}^{\mathbb{R}}\right)_{b(4 l+j)}^{t}=\sum_{m=1}^{n} \bar{\partial}_{q_{m}^{\prime}} \cdot \overline{\mathbf{a}}_{(l+1) m},
\end{aligned}
$$

by $\left(\mathbf{a}^{\mathbb{R}}\right)^{t}=\left(\overline{\mathbf{a}}^{t}\right)^{\mathbb{R}}$, which can be proved as [38, Lemma 2.1 (1)]. The proposition is proved.

Corollary 2.2 Let $\bar{Q}_{l+1}:=X_{4 l+1}+\mathbf{i} X_{4 l+2}+\mathbf{j} X_{4 l+3}+\mathbf{k} X_{4 l+4}$. Then, $R_{\mathbf{a} *}\left(\bar{Q}_{1}, \ldots, \bar{Q}_{n}\right)=$ $\left(\bar{Q}_{1}, \ldots, \bar{Q}_{n}\right) \overline{\mathbf{a}}^{t}$, for rotation $R_{\mathbf{a}}$ in (2.34) with $\mathbf{a} \in \operatorname{Sp}(n)$.

Since $\bar{Q}_{l}=\bar{\partial}_{q l}$ at the origin of $\mathscr{H}$, the above identity holds at the origin by Proposition 2.2. It holds at other places by the left invariance. By applying the representation $\tau$ in (2.13), i.e., $\tau\left(q_{1} q_{2}\right)=\tau\left(q_{1}\right) \tau\left(q_{2}\right)$ for any $q_{1}, q_{2} \in \mathbb{H}$ (cf. [33, Proposition 2.1]), we get

$$
\begin{aligned}
& R_{\mathbf{a} *}\left(\begin{array}{llllll}
Z_{00^{\prime}} & Z_{01^{\prime}} & \cdots & Z_{(2 l) 0^{\prime}} & Z_{(2 l) 1^{\prime}} & \cdots \\
Z_{10^{\prime}} & Z_{11^{\prime}} & \cdots & Z_{(2 l+1) 0^{\prime}} & Z_{(2 l+1) 1^{\prime}} & \ldots
\end{array}\right)
\end{aligned}
$$

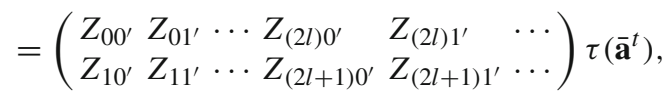

where $\tau\left(\overline{\mathbf{a}}^{t}\right)$ is a $(2 n) \times(2 n)$ complex matrix with $\overline{\mathbf{a}}_{j k}$ replaced by the $2 \times 2$ matrix $\tau\left(\overline{\mathbf{a}}_{j k}\right)$. (2.46) implies that each column in (2.14) is not preserved under rotations (2.34) of $\mathrm{Sp}(n)$. The commutativity (2.19) of each column that plays a very important role in the construction of our complexes (1.4) is destroyed. So by definition (2.23), (2.26) and (2.27), the differential operators $\mathscr{D}_{j}$ 's in the complex (1.4) in terms of $Z_{A}^{A^{\prime}}$ 's are not invariant under $\operatorname{Sp}(n)$. Therefore, they are not invariant under $\operatorname{Sp}(2(n+2), \mathbb{C})$.

Another difference is that the kernel of the tangential $k$-Cauchy-Fueter in space of $L^{2}$ integrable function on the left quaternionic Heisenberg group $\widetilde{\mathscr{H}}$ is infinite dimensional [32], while it is trivial on the right quaternionic Heisenberg group $\mathscr{H}$, since such a function satisfies $\Delta_{b} f=0$ by Proposition 2.4 and $\operatorname{ker} \Delta_{b}=\{0\}$ in the $L^{2}$ space.

On the other hand, if we choose the complex horizontal fields on $\mathscr{H}$

$$
\left(\widehat{Z}_{A A^{\prime}}\right):=\left(\begin{array}{cc}
-Y_{1}+\mathbf{i} Y_{2} & -Y_{3}-\mathbf{i} Y_{4} \\
Y_{3}-\mathbf{i} Y_{4} & -Y_{1}-\mathbf{i} Y_{2} \\
\vdots & \vdots \\
-Y_{4 l+1}+\mathbf{i} Y_{4 l+2} & -Y_{4 l+3}-\mathbf{i} Y_{4 l+4} \\
Y_{4 l+3}-\mathbf{i} Y_{4 l+4} & -Y_{4 l+1}-\mathbf{i} Y_{4 l+2} \\
\vdots & \vdots
\end{array}\right)
$$

with $Y_{4 l+1}$ replaced by $-Y_{4 l+1}$, then $\widehat{Z}_{A A^{\prime}}$ 's satisfy

$$
\begin{aligned}
& {\left[\widehat{Z}_{(2 l) 0^{\prime}}, \widehat{Z}_{(2 l+1) 0^{\prime}}\right]=8\left(\partial_{t_{2}}-\mathbf{i} \partial_{t_{3}}\right),} \\
& {\left[\widehat{Z}_{(2 l) 1^{\prime}}, \widehat{Z}_{(2 l+1) 1^{\prime}}\right]=8\left(\partial_{t_{2}}+\mathbf{i} \partial_{t_{3}}\right),} \\
& {\left[\widehat{Z}_{(2 l) 0^{\prime}}, \widehat{Z}_{(2 l+1) 1^{\prime}}\right]=\left[\widehat{Z}_{(2 l) 1^{\prime}}, \widehat{Z}_{(2 l+1) 0^{\prime}}\right]=-8 \mathbf{i} \partial_{t_{1}},}
\end{aligned}
$$


$l=0, \ldots, n-1$, and any other bracket vanishes, i.e., we can embed the real Lie algebra of $\mathscr{H}$ into the complex Lie algebra $\mathfrak{u}_{-}$. Then, the complexes (2.39) on $\mathrm{U}_{-}$induce a family of complexes on $\mathscr{H}$ invariant under $\operatorname{Sp}(2(n+2), \mathbb{C})$. But the first operator is different from the first one in (1.4). Moreover, the $(n-1)$ th operator in the complex induced from $U_{-}$is a linear combination of $T_{\beta}$ 's (cf. [37, Proposition 4.3.3]), while the (n-1)th operator in (1.4) involves only $Z_{A A^{\prime}}$ 's. So we get two different families of complexes on $\mathscr{H}$. Here, changing $Y_{4 l+1}$ to $-Y_{4 l+1}$ corresponds to changing the sign before $x_{4 l+1}^{2}$ in the defining function (1.2) of the hypersurface $\mathcal{S}$. The resulting hypersurface is essentially the boundary of the quaternionic Siegel domain.

On other quadratic hypersurface, there is no reason to expect that the restriction of the $k$-Cauchy-Fueter operators and complexes is invariant in general under the action of $\operatorname{Sp}(n)$.

\subsection{The adjoint operator}

On a domain $\Omega \subset \mathscr{H}$, denote the inner product

$$
(u, v):=\int_{\Omega} u \cdot \bar{v} \mathrm{~d} V,
$$

for $u, v \in L^{2}(\Omega, \mathbb{C})$, where $\mathrm{d} V$ is the Lebesgue measure on $\mathscr{H}$. The inner product of $L^{2}\left(\Omega, \mathscr{V}_{1}\right)$ is defined as

$$
\langle f, h\rangle:=\sum_{A=0}^{2 n-1} \sum_{A_{2}^{\prime}, \ldots, A_{k}^{\prime}=0^{\prime}, 1^{\prime}}\left(f_{A A_{2}^{\prime} \ldots A_{k}^{\prime}}, h_{A A_{2}^{\prime} \ldots A_{k}^{\prime}}\right)
$$

for $f, h \in L^{2}\left(\Omega, \mathscr{V}_{1}\right)$, and $\|f\|:=\langle f, f\rangle^{\frac{1}{2}}$. We define inner products of $L^{2}\left(\Omega, \mathscr{V}_{0}\right)$ and $L^{2}\left(\Omega, \mathscr{V}_{2}\right)$ similarly. Define the $L^{2}$-norm on $\mathscr{H} / \mathscr{H}_{\mathbb{Z}}$ by

$$
\|f\|_{L^{2}\left(\mathscr{H} / \mathscr{H}_{\mathbb{Z}}\right)}^{2}=\|f\|_{L^{2}(\mathscr{F})}^{2}=\int_{\mathscr{F}}|f|^{2} \mathrm{~d} V .
$$

Proposition 2.3 The formal adjoint operator of $Z_{A}^{A^{\prime}}$ is

$$
\left(Z_{A}^{A^{\prime}}\right)^{*}=\delta_{A^{\prime}}^{A}, \quad \text { where } \delta_{A^{\prime}}^{A}:=-\overline{Z_{A}^{A^{\prime}}} .
$$

Proof For $u, v \in C_{0}^{\infty}(\mathscr{H}, \mathbb{C})$, we have

$$
\left(Y_{a} u, v\right)=\left(u,-Y_{a} v\right)
$$

by integration by part. So $\left(\left(Y_{a} \pm \mathbf{i} Y_{b}\right) u, v\right)=\left(u,-\left(Y_{a} \mp \mathbf{i} Y_{b}\right) v\right)$. Then, (2.48) holds since $Z_{A}^{A^{\prime}}$ has the form $Y_{a} \pm \mathbf{i} Y_{b}$ for some $a$ and $b$ by (2.16). Thus, we have

$$
\left(Z_{A}^{A^{\prime}} u, v\right)=\left(u, \delta_{A^{\prime}}^{A} v\right)
$$

over $\mathscr{H}$. For (2.49) over $\mathscr{H} / \mathscr{H}_{\mathbb{Z}}$, by using the unit partition, it is sufficient to show it for $v \in C_{0}^{\infty}(\mathscr{H}, \mathbb{C})$. This case follows from the result over $\mathscr{H}$.

Lemma 2.3 For $f \in C_{0}^{1}\left(\mathscr{H}, \mathscr{V}_{1}\right)$ or $C^{1}\left(\mathscr{H} / \mathscr{H}_{\mathbb{Z}}, \mathscr{V}_{1}\right)$, we have

$$
\left(\mathscr{D}_{0}^{*} f\right)_{A_{1}^{\prime} \ldots A_{k}^{\prime}}=\sum_{A=0}^{2 n-1} \delta_{\left(A_{1}^{\prime}\right.}^{A} f_{\left.A_{2}^{\prime} \ldots A_{k}^{\prime}\right) A} .
$$


Proof The proof is similar to that for the $k$-Cauchy-Fueter operator over $\mathbb{H}^{n}$ (cf. [40, Lemma 3.1]). For any $g \in C^{1}\left(\mathscr{H} / \mathscr{H}_{\mathbb{Z}}, \mathscr{V}_{0}\right)$, we have

$$
\begin{aligned}
\left\langle\mathscr{D}_{0} g, f\right\rangle & =\sum_{A, A_{2}^{\prime}, \ldots, A_{k}^{\prime}}\left(\sum_{A_{1}^{\prime}} Z_{A}^{A_{1}^{\prime}} g_{A_{1}^{\prime} \ldots A_{k}^{\prime}}, f_{A_{2}^{\prime} \ldots A_{k}^{\prime} A}\right)=\sum_{A, A_{1}^{\prime}, \ldots, A_{k}^{\prime}}\left(g_{A_{1}^{\prime} \ldots A_{k}^{\prime}}, \delta_{A_{1}^{\prime}}^{A} f_{A_{2}^{\prime} \ldots A_{k}^{\prime} A}\right) \\
& =\sum_{A_{1}^{\prime}, \ldots, A_{k}^{\prime}}\left(g_{A_{1}^{\prime} \ldots A_{k}^{\prime}}, \sum_{A} \delta_{\left(A_{1}^{\prime}\right.}^{A} f_{\left.A_{2}^{\prime} \ldots A_{k}^{\prime}\right) A}\right)=\left\langle g, \mathscr{D}_{0}^{*} f\right\rangle
\end{aligned}
$$

by using (2.49) and symmetrization

$$
\sum_{A_{1}^{\prime}, \ldots, A_{k}^{\prime}}\left(g_{A_{1}^{\prime} \ldots A_{k}^{\prime}}, G_{A_{1}^{\prime} \ldots A_{k}^{\prime}}\right)=\sum_{A_{1}^{\prime}, \ldots, A_{k}^{\prime}}\left(g_{A_{1}^{\prime} \ldots A_{k}^{\prime}}, G_{\left(A_{1}^{\prime} \ldots A_{k}^{\prime}\right)}\right)
$$

for any $g \in L^{2}\left(\mathscr{H}, \odot^{k} \mathbb{C}^{2}\right), G \in L^{2}\left(\mathscr{H}, \otimes^{k} \mathbb{C}^{2}\right)$. (cf. [40, (3.4)]). Here, we have to symmetrise the primed indices in $\sum_{A} \delta_{A_{1}^{\prime}}^{A} f_{A_{2}^{\prime} \ldots A_{k}^{\prime} A}$ since only after symmetrization it becomes an element of $C_{0}^{1}\left(\mathscr{H}, \mathscr{V}_{0}\right)$.

$\mathscr{D}_{0}^{*} \mathscr{D}_{0}$ is simple since it is diagonal by the following proposition.

Proposition 2.4 For $f \in C^{2}\left(\Omega, \mathscr{V}_{0}\right)$, we have

$$
\mathscr{D}_{0}^{*} \mathscr{D}_{0} f=\Delta_{b} f .
$$

Proof Recall that for a $\otimes^{k} \mathbb{C}^{2}$-valued function $F_{A_{1}^{\prime} \ldots A_{k}^{\prime}}$ symmetric in $A_{2}^{\prime} \ldots A_{k}^{\prime}$, we have

$$
F_{\left(A_{1}^{\prime} \ldots A_{k}^{\prime}\right)}=\frac{1}{k}\left(F_{A_{1}^{\prime} A_{2}^{\prime} \ldots A_{k}^{\prime}}+\cdots+F_{A_{s}^{\prime} A_{1}^{\prime} \ldots \widehat{A_{s}^{\prime}} \ldots A_{k}^{\prime}}+\cdots+F_{A_{k}^{\prime} A_{1}^{\prime} \ldots \widehat{A_{k}^{\prime}}}\right),
$$

by the definition of symmetrization (2.17). As usual, a hat means omittance of the corresponding index. Then, for fixed $A_{1}^{\prime}, \ldots, A_{k}^{\prime}=0^{\prime}, 1^{\prime}$,

$$
\begin{aligned}
\left(\mathscr{D}_{0}^{*} \mathscr{D}_{0} f\right)_{A_{1}^{\prime} \ldots A_{k}^{\prime}} & =\sum_{A} \delta_{\left(A_{1}^{\prime}\right.}^{A}\left(\mathscr{D}_{0} f\right)_{\left.A_{2}^{\prime} \ldots A_{k}^{\prime}\right) A}=\frac{1}{k} \sum_{s=1}^{k} \delta_{A_{s}^{\prime}}^{A}\left(\mathscr{D}_{0} f\right)_{\ldots} \widehat{A_{s}^{\prime} \ldots A_{k}^{\prime} A} \\
& =-\frac{1}{k} \sum_{s=1}^{k} \sum_{A, A^{\prime}} \overline{Z_{A}^{A_{s}^{\prime}}} Z_{A}^{A^{\prime}} f_{A^{\prime} \ldots \widehat{A_{s}^{\prime}} \ldots A_{k}^{\prime}} \\
& =\frac{1}{k} \sum_{s=1}^{k} \sum_{A^{\prime}} \Delta_{b} f_{A^{\prime} \ldots \widehat{A_{s}^{\prime}} \ldots A_{k}^{\prime}} \delta_{A_{s}^{\prime} A^{\prime}}=\Delta_{b} f_{A_{1}^{\prime} \ldots A_{k}^{\prime}},
\end{aligned}
$$

by using the following Lemma 2.4 and $f$ symmetric in the primed indices, where $\mathscr{D}_{0}^{*}$ is given by (2.50). The proposition is proved.

Lemma 2.4 For $A^{\prime}, B^{\prime}=0^{\prime}, 1^{\prime}$, we have

$$
\sum_{A=0}^{2 n-1} \overline{Z_{A}^{A^{\prime}}} Z_{A}^{B^{\prime}}=-\delta_{A^{\prime} B^{\prime}} \Delta_{b}
$$


Proof Note that

$$
\begin{aligned}
\overline{Z_{2 l}^{0^{\prime}}} Z_{2 l}^{0^{\prime}}+\overline{Z_{2 l+1}^{0^{\prime}}} Z_{2 l+1}^{0^{\prime}}= & \left(-Y_{4 l+3}+\mathbf{i} Y_{4 l+4}\right)\left(-Y_{4 l+3}-\mathbf{i} Y_{4 l+4}\right) \\
& +\left(Y_{4 l+1}+\mathbf{i} Y_{4 l+2}\right)\left(Y_{4 l+1}-\mathbf{i} Y_{4 l+2}\right) \\
= & \sum_{k=1}^{4} Y_{4 l+k}^{2}+\mathbf{i}\left[Y_{4 l+3}, Y_{4 l+4}\right]-\mathbf{i}\left[Y_{4 l+1}, Y_{4 l+2}\right]=\sum_{k=1}^{4} Y_{4 l+k}^{2},
\end{aligned}
$$

by (2.21), whose summation over $l$ gives us (2.53) for $A^{\prime}=B^{\prime}=0^{\prime}$. Similarly, we have

$$
\begin{aligned}
\overline{Z_{2 l}^{0^{\prime}}} Z_{2 l}^{1^{\prime}}+\overline{Z_{2 l+1}^{0^{\prime}}} Z_{2 l+1}^{1^{\prime}}= & \left(-Y_{4 l+3}+\mathbf{i} Y_{4 l+4}\right)\left(-Y_{4 l+1}-\mathbf{i} Y_{4 l+2}\right) \\
& +\left(Y_{4 l+1}+\mathbf{i} Y_{4 l+2}\right)\left(-Y_{4 l+3}+\mathbf{i} Y_{4 l+4}\right) \\
= & -\left[Y_{4 l+1}, Y_{4 l+3}\right]-\left[Y_{4 l+2}, Y_{4 l+4}\right] \\
& +\mathbf{i}\left[Y_{4 l+1}, Y_{4 l+4}\right]-\mathbf{i}\left[Y_{4 l+2}, Y_{4 l+3}\right]=0,
\end{aligned}
$$

by (2.21), whose summation over $l$ gives us (2.53) for $A^{\prime}=0, B^{\prime}=1^{\prime}$. Similarly, (2.53) holds for $A^{\prime}=1, B^{\prime}=0^{\prime}$ and $A^{\prime}=B^{\prime}=1^{\prime}$ by

$$
\begin{aligned}
\overline{Z_{2 l}^{1^{\prime}}} Z_{2 l}^{0^{\prime}}+\overline{Z_{2 l+1}^{1^{\prime}}} Z_{2 l+1}^{0^{\prime}}= & {\left[Y_{4 l+1}, Y_{4 l+3}\right]+\left[Y_{4 l+2}, Y_{4 l+4}\right] } \\
& +\mathbf{i}\left[Y_{4 l+1}, Y_{4 l+4}\right]-\mathbf{i}\left[Y_{4 l+2}, Y_{4 l+3}\right]=0, \\
\overline{Z_{2 l}^{1^{\prime}}} Z_{2 l}^{1^{\prime}}+\overline{Z_{2 l+1}^{1^{\prime}}} Z_{2 l+1}^{1^{\prime}}= & \sum_{k=1}^{4} Y_{4 l+k}^{2}+\mathbf{i}\left[Y_{4 l+1}, Y_{4 l+2}\right]-\mathbf{i}\left[Y_{4 l+3}, Y_{4 l+4}\right]=\sum_{k=1}^{4} Y_{4 l+k}^{2} .
\end{aligned}
$$

Then, (2.53) follows.

\section{The $L^{2}$ estimate}

We begin with the following Poincaré-type inequality, which was proved for general vector fields satisfying Hörmander's condition (cf. [20, Theorem 2.1]). So it holds over $\mathscr{H}$.

Proposition 3.1 (Poincaré-type inequality) For each $f$ with $\sum_{a=1}^{4 n}\left|Y_{a} f\right|^{2} \in L^{1}(\mathscr{H})$, we have

$$
\int_{B_{r}}\left|f-f_{B_{r}}\right|^{2} \mathrm{~d} V \leq C r^{2} \int_{B_{r}} \sum_{a=1}^{4 n}\left|Y_{a} f\right|^{2} \mathrm{~d} V,
$$

where $B_{r}$ is a ball of radius $r$ and $f_{B_{r}}=\int_{B_{r}} f \mathrm{~d} V / \int_{B_{r}} \mathrm{~d} V$.

We say $f \in L^{2}\left(\mathscr{H} / \mathscr{H}_{\mathbb{Z}}, \mathscr{V}_{1}\right)$ satisfies $f \perp$ constant vectors if $\langle f, C\rangle=0$ for any constant vector $C \in \mathscr{V}_{1}$.

Lemma 3.1 There exists some $c>0$ such that

$$
\left\langle\Delta_{b} f, f\right\rangle \geq c\|f\|_{L^{2}\left(\mathscr{H} / \mathscr{H}_{\mathbb{Z}}\right)}^{2},
$$

for $f \in C^{2}\left(\mathscr{H} / \mathscr{H}_{\mathbb{Z}}, \mathscr{V}_{1}\right)$ and $f \perp$ constant vectors. 
Proof As $\bigcup_{(n, m) \in \mathscr{H}_{\mathbb{Z}}} \tau_{(n, m)} \mathscr{F}=\mathscr{H}$ by Proposition 2.1, we can choose some $r>0$ and a finite number of elements $\left(n_{i}, m_{i}\right) \in \mathscr{H}_{\mathbb{Z}}, i=1, \ldots, N$, such that

$$
\mathscr{F} \subset B_{r} \subset \bigcup_{i=1}^{N} \tau_{\left(n_{i}, m_{i}\right)} \mathscr{F} .
$$

Recall that if we identify $f \in C^{2}\left(\mathscr{H} / \mathscr{H}_{\mathbb{Z}}, \mathscr{V}_{1}\right)$ with a periodic function on $\mathscr{H}$, so is $Y_{a} f$. Then, the Poincaré-type inequality (3.1) implies that

$$
N \sum_{a=1}^{4 n}\left\|Y_{a} f\right\|_{L^{2}(\mathscr{F})}^{2} \geq \sum_{a=1}^{4 n}\left\|Y_{a} f\right\|_{L^{2}\left(B_{r}\right)}^{2} \geq \frac{1}{C r^{2}} \int_{B_{r}}\left|f-f_{B_{r}}\right|^{2} \mathrm{~d} V \geq \frac{1}{C r^{2}}\left\|f-f_{B_{r}}\right\|_{L^{2}(\mathscr{F})}^{2} .
$$

Since $f \perp$ constant vectors, we have

$$
\left\|f-f_{B_{r}}\right\|_{L^{2}\left(\mathscr{H}^{\prime} / \mathscr{H}_{\mathbb{Z}}\right)}^{2}=\|f\|_{L^{2}\left(\mathscr{H}^{\prime} / \mathscr{C}_{\mathbb{Z}}\right)}^{2}+\left\|f_{B_{r}}\right\|_{L^{2}\left(\mathscr{H}^{\prime} / \mathscr{H}_{\mathbb{Z}}\right)}^{2} \geq\|f\|_{L^{2}\left(\mathscr{H}^{\prime} / \mathscr{H}_{\mathbb{Z}}\right)}^{2} .
$$

Thus, we find that

$$
\left\langle\Delta_{b} f, f\right\rangle \geq c\left\|f-f_{B_{r}}\right\|_{L^{2}\left(\mathscr{H} \mid \mathscr{H}_{\mathbb{Z}}\right)}^{2} \geq c\|f\|_{L^{2}\left(\mathscr{H}^{\prime} \mid \mathscr{H}_{\mathbb{Z}}\right)}^{2},
$$

for constant $c=\frac{1}{N C r^{2}}$.

Lemma 3.2 (cf. [40, Lemma 2.1]) For any $h, H \in \mathbb{C}^{2 n} \otimes \mathbb{C}^{2 n}$, we have

$$
\sum_{A, B} h_{B A} \overline{H_{A B}}=\sum_{A, B} h_{A B} \overline{H_{A B}}-2 \sum_{A, B} h_{[A B]} \overline{H_{[A B]}} .
$$

We have the following $L^{2}$ estimate.

Theorem 3.1 For $n>3, k \geq 2$, there exists some $c_{n, k}>0$ such that

$$
\left\|\mathscr{D}_{0}^{*} f\right\|^{2}+\left\|\mathscr{D}_{1} f\right\|^{2} \geq c_{n, k}\|f\|^{2},
$$

for $f \in \operatorname{Dom}\left(\mathscr{D}_{1}\right) \cap \operatorname{Dom}\left(\mathscr{D}_{0}^{*}\right)$ and $f \perp$ constant vectors over $\mathscr{H} / \mathscr{H} \mathbb{Z}$.

Proof We use the $L^{2}$ method for the $k$-Cauchy-Fueter operator on $\mathbb{H}^{n}$ in [40]. Since $C^{2}$ functions are dense in $\operatorname{Dom}\left(\mathscr{D}_{1}\right) \cap \operatorname{Dom}\left(\mathscr{D}_{0}^{*}\right)$ for the compact manifold $\mathscr{H} / \mathscr{H}_{\mathbb{Z}}$, it is sufficient to prove (3.2) for $f \in C^{2}\left(\mathscr{H} / \mathscr{H}_{\mathbb{Z}}, \odot^{k-1} \mathbb{C}^{2} \otimes \mathbb{C}^{2 n}\right)$. We have

$$
\begin{aligned}
k\left\langle\mathscr{D}_{0}^{*} f, \mathscr{D}_{0}^{*} f\right\rangle= & k\left\langle\mathscr{D}_{0} \mathscr{D}_{0}^{*} f, f\right\rangle=k \sum_{B, A_{2}^{\prime}, \ldots, A_{k}^{\prime}}\left(\sum_{A_{1}^{\prime}} Z_{B}^{A_{1}^{\prime}} \sum_{A} \delta_{\left(A_{1}^{\prime}\right.}^{A} f_{\left.A_{2}^{\prime} \ldots A_{k}^{\prime}\right) A}, f_{A_{2}^{\prime} \ldots A_{k}^{\prime} B}\right) \\
= & \sum_{A, B, A_{1}^{\prime}, \ldots, A_{k}^{\prime}}\left(Z_{B}^{A_{1}^{\prime}} \delta_{A_{1}^{\prime}}^{A} f_{A_{2}^{\prime} \ldots A_{k}^{\prime} A}, f_{A_{2}^{\prime} \ldots A_{k}^{\prime} B}\right) \\
& +\sum_{A, B, A_{1}^{\prime}, \ldots, A_{k}^{\prime}} \sum_{s=2}^{k}\left(Z_{B}^{A_{1}^{\prime}} \delta_{A_{s}^{\prime}}^{A} f_{A_{1}^{\prime} \ldots A_{s}^{\prime} \ldots A_{k}^{\prime} A}, f_{A_{2}^{\prime} \ldots A_{k}^{\prime} B}\right)=: \Sigma_{0}+\Sigma_{1},
\end{aligned}
$$

by using (2.51) to expand the symmetrization. Note that

$$
\Sigma_{0}=\sum_{A_{1}^{\prime}, \ldots, A_{k}^{\prime}}\left(\sum_{A} \delta_{A_{1}^{\prime}}^{A} f_{A_{2}^{\prime} \ldots A_{k}^{\prime} A}, \sum_{B} \delta_{A_{1}^{\prime}}^{B} f_{A_{2}^{\prime} \ldots A_{k}^{\prime} B}\right)=\sum_{A_{1}^{\prime}, \ldots, A_{k}^{\prime}}\left\|\sum_{A} \delta_{A_{1}^{\prime}}^{A} f_{A_{2}^{\prime} \ldots A_{k}^{\prime} A}\right\|^{2} \geq 0,
$$


and

$$
\begin{aligned}
\Sigma_{1}= & \sum_{s=2}^{k} \sum_{A, B, A_{1}^{\prime}, \ldots, A_{k}^{\prime}}\left(\delta_{A_{s}^{\prime}}^{A} Z_{B}^{A_{1}^{\prime}} f_{A_{1}^{\prime} \ldots \widehat{A_{s}^{\prime}} \ldots A_{k}^{\prime} A}, f_{A_{2}^{\prime} \ldots A_{k}^{\prime} B}\right) \\
& +\sum_{s=2}^{k} \sum_{A, B, A_{1}^{\prime}, \ldots, A_{k}^{\prime}}\left(\left[Z_{B}^{A_{1}^{\prime}}, \delta_{A_{s}^{\prime}}^{A}\right] f_{A_{1}^{\prime} \ldots \widehat{A_{s}^{\prime}} \ldots A_{k}^{\prime} A}, f_{A_{2}^{\prime} \ldots A_{k}^{\prime} B}\right)=: \Sigma_{11}+\mathscr{C}
\end{aligned}
$$

by using commutators. For the first sum, we have

$$
\begin{aligned}
\Sigma_{11} & =\sum_{s=2}^{k} \sum_{A, B, A_{1}^{\prime}, \ldots, A_{k}^{\prime}}\left(Z_{B}^{A_{1}^{\prime}} f_{A_{1}^{\prime} \ldots \widehat{A_{s}^{\prime}} \ldots A_{k}^{\prime} A}, Z_{A}^{A_{s}^{\prime}} f_{A_{2}^{\prime} \ldots A_{k}^{\prime} B}\right) \\
& =\sum_{s=2}^{k} \sum_{A, B} \sum_{\widehat{A_{1}^{\prime}}, \ldots, \widehat{A_{s}^{\prime}}, \ldots, A_{k}^{\prime}}\left(\sum_{A_{1}^{\prime}} Z_{B}^{A_{1}^{\prime}} f_{A_{1}^{\prime} \ldots \widehat{A_{s}^{\prime}} \ldots A_{k}^{\prime} A}, \sum_{A_{s}^{\prime}} Z_{A}^{A_{s}^{\prime}} f_{\left.A_{s}^{\prime} A_{2}^{\prime} \ldots \widehat{A_{s}^{\prime}} \ldots A_{k}^{\prime} B\right)} \sum_{B_{3}^{\prime}, \ldots, B_{k}^{\prime}=0^{\prime}, 1^{\prime}}\left(\sum_{A, B} Z_{B}^{A^{\prime}} f_{A A^{\prime} B_{3}^{\prime} \ldots B_{k}^{\prime}}, \sum_{A^{\prime}} Z_{A}^{A^{\prime}} f_{B A^{\prime} B_{3}^{\prime} \ldots B_{k}^{\prime}}\right)\right.
\end{aligned}
$$

by relabeling indices and $f$ symmetric in the primed indices. Then, by applying Lemma 3.2 with $h_{B A}=\sum_{A^{\prime}} Z_{B}^{A^{\prime}} f_{A A^{\prime} B_{3}^{\prime} \ldots B_{k}^{\prime}}$ and $H_{A B}=\sum_{A^{\prime}} Z_{A}^{A^{\prime}} f_{B A^{\prime} B_{3}^{\prime} \ldots B_{k}^{\prime}}$ for fixed $B_{3}^{\prime}, \ldots, B_{k}^{\prime}$, we get

$$
\begin{aligned}
\Sigma_{11} & =(k-1) \sum_{B_{3}^{\prime}, \ldots, B_{k}^{\prime}} \sum_{A, B}\left(\left\|\sum_{A^{\prime}} Z_{A}^{A^{\prime}} f_{B A^{\prime} B_{3}^{\prime} \ldots B_{k}^{\prime}}\right\|^{2}-2\left\|\sum_{A^{\prime}} Z_{[A}^{A^{\prime}} f_{B] A^{\prime} B_{3}^{\prime} \ldots B_{k}^{\prime}}\right\|^{2}\right) \\
& =(k-1) \sum_{B_{3}^{\prime}, \ldots, B_{k}^{\prime}} \sum_{A, B}\left\|\sum_{A^{\prime}} Z_{A}^{A^{\prime}} f_{B A^{\prime} B_{3}^{\prime} \ldots B_{k}^{\prime}}\right\|^{2}-\frac{k-1}{2}\left\|\mathscr{D}_{1} f\right\|^{2},
\end{aligned}
$$

where

$$
\begin{aligned}
& \sum_{A}\left\|\sum_{A^{\prime}} Z_{A}^{A^{\prime}} f_{B A^{\prime} B_{3}^{\prime} \ldots B_{k}^{\prime}}\right\|^{2}=\sum_{A, A^{\prime}, B^{\prime}}\left(Z_{A}^{A^{\prime}} f_{B A^{\prime} B_{3}^{\prime} \ldots B_{k}^{\prime}}, Z_{A}^{B^{\prime}} f_{B B^{\prime} B_{3}^{\prime} \ldots B_{k}^{\prime}}\right) \\
& =\sum_{A^{\prime}, B^{\prime}}\left(-\sum_{A} \overline{Z_{A}^{B^{\prime}}} Z_{A}^{A^{\prime}} f_{B A^{\prime} B_{3}^{\prime} \ldots B_{k}^{\prime}}, f_{B B^{\prime} B_{3}^{\prime} \ldots B_{k}^{\prime}}\right)=\sum_{B^{\prime}}\left(\Delta_{b} f_{B B^{\prime} B_{3}^{\prime} \ldots B_{k}^{\prime}}, f_{B B^{\prime} B_{3}^{\prime} \ldots B_{k}^{\prime}}\right)
\end{aligned}
$$

by Lemma 2.4. Thus, by substituting (3.4)-(3.5) and (3.7)-(3.8) to (3.3), we get

$$
k\left\|\mathscr{D}_{0}^{*} f\right\|^{2}+\frac{k-1}{2}\left\|\mathscr{D}_{1} f\right\|^{2} \geq(k-1)\left\langle\Delta_{b} f, f\right\rangle+\mathscr{C} .
$$

To control the commutator term $\mathscr{C}$ in (3.5), note that

$$
\overline{Z_{2 l}^{0^{\prime}}}=Z_{2 l+1}^{1^{\prime}}, \quad \overline{Z_{2 l}^{1^{\prime}}}=-Z_{2 l+1}^{0^{\prime}}
$$

by (2.16). Then, it follows from Lemma 2.1 that (1)

$$
\left[Z_{A}^{A^{\prime}}, \overline{Z_{B}^{B^{\prime}}}\right]=0, \text { for } A^{\prime} \neq B^{\prime},
$$


$A, B=0, \ldots, 2 n-1 ;(2)$ for $A^{\prime}=B^{\prime}$, we have

$$
\begin{aligned}
& {\left[Z_{2 l}^{0^{\prime}}, \overline{Z_{2 l+1}^{0^{\prime}}}\right]=\left[Z_{2 l}^{1^{\prime}}, \overline{Z_{2 l+1}^{1^{\prime}}}\right]=-8\left(\partial_{s_{2}}+\mathbf{i} \partial_{s_{3}}\right),} \\
& {\left[Z_{2 l+1}^{0^{\prime}}, \overline{Z_{2 l}^{0^{\prime}}}\right]=\left[Z_{2 l+1}^{1^{\prime}}, \overline{Z_{2 l}^{1^{\prime}}}\right]=8\left(\partial_{s_{2}}-\mathbf{i} \partial_{s_{3}}\right),} \\
& \quad\left[Z_{2 l}^{0^{\prime}}, \overline{Z_{2 l}^{0^{\prime}}}\right]=\left[Z_{2 l}^{1^{\prime}}, \overline{Z_{2 l}^{1^{\prime}}}\right]=-\left[Z_{2 l+1}^{0^{\prime}}, \overline{Z_{2 l+1}^{0^{\prime}}}\right]=-\left[Z_{2 l+1}^{1^{\prime}}, \overline{Z_{2 l+1}^{1^{\prime}}}\right]=8 \mathbf{i} \partial_{s_{1}}
\end{aligned}
$$

(3) if $\{A, B\} \neq\{2 l, 2 l+1\}$ for any $l$, then $\left[Z_{A}^{A^{\prime}}, \overline{Z_{B}^{B^{\prime}}}\right]=0$ for any $A^{\prime}, B^{\prime}$. Thus, we have

$$
\begin{aligned}
\mathscr{C}= & \sum_{s=2}^{k} \sum_{A, B, A_{1}^{\prime}, \ldots, A_{k}^{\prime}}\left(\left[Z_{B}^{A_{1}^{\prime}}, \overline{Z_{A}^{A_{s}^{\prime}}}\right] f_{A_{1}^{\prime} \ldots \widehat{A_{s}^{\prime}} \ldots A_{k}^{\prime} A}, f_{A_{2}^{\prime} \ldots A_{k}^{\prime} B}\right) \\
= & -(k-1) \sum_{A, B, B^{\prime}, B_{3}^{\prime}, \ldots, B_{k}^{\prime}}\left(\left[Z_{B}^{B^{\prime}}, \overline{Z_{A}^{B^{\prime}}}\right] f_{B^{\prime} B_{3}^{\prime} \ldots B_{k}^{\prime} A}, f_{B^{\prime} B_{3}^{\prime} \ldots B_{k}^{\prime} B}\right), \\
= & -(k-1) \sum_{B^{\prime}, B_{3}^{\prime}, \ldots, B_{k}^{\prime}} \sum_{l=0}^{n-1}\left\{\left(\left[Z_{2 l}^{B^{\prime}}, \overline{Z_{2 l}^{B^{\prime}}}\right] f_{B^{\prime} B_{3}^{\prime} \ldots B_{k}^{\prime}(2 l)}, f_{B^{\prime} B_{3}^{\prime} \ldots B_{k}^{\prime}(2 l)}\right)\right. \\
& +\left(\left[Z_{2 l}^{B^{\prime}}, \overline{Z_{2 l+1}^{B^{\prime}}}\right] f_{B^{\prime} B_{3}^{\prime} \ldots B_{k}^{\prime}(2 l+1)}, f_{B^{\prime} B_{3}^{\prime} \ldots B_{k}^{\prime}(2 l)}\right) \\
& +\left(\left[Z_{2 l+1}^{B^{\prime}}, \overline{Z_{2 l}^{B^{\prime}}}\right] f_{B^{\prime} B_{3}^{\prime} \ldots B_{k}^{\prime}(2 l)}, f_{B^{\prime} B_{3}^{\prime} \ldots B_{k}^{\prime}(2 l+1)}\right) \\
& \left.+\left(\left[Z_{2 l+1}^{B^{\prime}}, \overline{Z_{2 l+1}^{B^{\prime}}}\right] f_{B^{\prime} B_{3}^{\prime} \ldots B_{k}^{\prime}(2 l+1)}, f_{B^{\prime} B_{3}^{\prime} \ldots B_{k}^{\prime}(2 l+1)}\right)\right\}
\end{aligned}
$$

by using (1) and (3) above, relabeling indices and $f$ symmetric in the primed indices. Apply (3.11) to $\mathscr{C}$ above to get

$$
\begin{aligned}
\mathscr{C}= & -8(k-1) \sum_{B^{\prime}, B_{3}^{\prime}, \ldots, B_{k}^{\prime}}\left\{\sum_{A=0}^{2 n-1}(-1)^{A}\left(\mathbf{i} \partial_{s_{1}} f_{B^{\prime} B_{3}^{\prime} \ldots B_{k}^{\prime} A}, f_{B^{\prime} B_{3}^{\prime} \ldots B_{k}^{\prime} A}\right)\right. \\
& +\sum_{l=0}^{n-1}\left(-\left(\partial_{s_{2}}+\mathbf{i} \partial_{s_{3}}\right) f_{B^{\prime} B_{3}^{\prime} \ldots B_{k}^{\prime}(2 l+1)}, f_{B^{\prime} B_{3}^{\prime} \ldots B_{k}^{\prime}(2 l)}\right) \\
& \left.+\sum_{l=0}^{n-1}\left(\left(\partial_{s_{2}}-\mathbf{i} \partial_{s_{3}}\right) f_{B^{\prime} B_{3}^{\prime} \ldots B_{k}^{\prime}(2 l)}, f_{B^{\prime} B_{3}^{\prime} \ldots B_{k}^{\prime}(2 l+1)}\right)\right\} .
\end{aligned}
$$

For any $u, v \in C^{1}\left(\mathscr{H} / \mathscr{H}_{\mathbb{Z}}, \mathbb{C}\right)$, we have

$$
8\left(\partial_{s_{1}} u, v\right)=-\frac{1}{n} \sum_{l=0}^{n-1}\left(\left[Y_{4 l+1}, Y_{4 l+2}\right] u+\left[Y_{4 l+3}, Y_{4 l+4}\right] u, v\right),
$$

by (2.21). As

$$
\begin{aligned}
\left|\left(\left[Y_{a}, Y_{b}\right] u, v\right)\right| & =\left|\left(Y_{b} u,-Y_{a} v\right)+\left(Y_{a} u, Y_{b} v\right)\right| \\
& \leq \frac{1}{2}\left(\left\|Y_{a} u\right\|^{2}+\left\|Y_{b} u\right\|^{2}+\left\|Y_{a} v\right\|^{2}+\left\|Y_{b} v\right\|^{2}\right),
\end{aligned}
$$


for $a, b=1, \ldots, 4 n$, we get

$$
\left|8\left(\partial_{s_{1}} u, v\right)\right| \leq \frac{1}{2 n} \sum_{a=1}^{4 n}\left(\left\|Y_{a} u\right\|^{2}+\left\|Y_{a} v\right\|^{2}\right) .
$$

Similarly, we have

$$
\left|\left(8\left(\partial_{s_{2}} \pm \mathbf{i} \partial_{s_{3}}\right) u, v\right)\right| \leq \frac{1}{n} \sum_{a=1}^{4 n}\left(\left\|Y_{a} u\right\|^{2}+\left\|Y_{a} v\right\|^{2}\right) .
$$

Then, apply (3.13)-(3.14) to the right-hand side of (3.12) to get

$$
|\mathscr{C}| \leq(k-1) \frac{3}{n} \sum_{A, B^{\prime}, B_{3}^{\prime}, \ldots, B_{k}^{\prime}} \sum_{a=1}^{4 n}\left\|Y_{a} f_{B^{\prime} B_{3}^{\prime} \ldots B_{k}^{\prime} A}\right\|^{2}=\frac{3(k-1)}{n}\left\langle\Delta_{b} f, f\right\rangle .
$$

So it follows from estimate (3.9) that

$$
k\left\|\mathscr{D}_{0}^{*} f\right\|^{2}+\frac{k-1}{2}\left\|\mathscr{D}_{1} f\right\|^{2} \geq(k-1)\left(1-\frac{3}{n}\right)\left\langle\Delta_{b} f, f\right\rangle .
$$

Now, by applying Lemma 3.1 we get (3.2).

\section{Hartogs' phenomenon}

\subsection{The nonhomogeneous tangential $\boldsymbol{k}$-Cauchy-Fueter equation over $\mathscr{H} / \mathscr{H}_{\mathbb{Z}}$}

Consider the Hilbert subspace $\mathcal{L}$ consisting of $f \in L^{2}\left(\mathscr{H} / \mathscr{H}_{\mathbb{Z}}, \mathscr{V}_{1}\right)$ and $f \perp$ constant vectors. The domain of $\square_{1}$ over $\mathcal{L}$ is

$\operatorname{Dom}\left(\square_{1}\right):=\left\{f \in \mathcal{L}: f \in \operatorname{Dom}\left(\mathscr{D}_{0}^{*}\right) \cap \operatorname{Dom}\left(\mathscr{D}_{1}\right), \mathscr{D}_{0}^{*} f \in \operatorname{Dom}\left(\mathscr{D}_{0}\right), \mathscr{D}_{1} f \in \operatorname{Dom}\left(\mathscr{D}_{1}^{*}\right)\right\}$.

Proposition 4.1 The associated Hodge-Laplacian $\square_{1}$ is densely defined, closed, self-adjoint and nonnegative operator on $\mathcal{L}$.

The proof is exactly the same as that of Proposition 3.1 in [40] since $\mathcal{L} \oplus$ \{const. $\}=$ $L^{2}\left(\mathscr{H} / \mathscr{H}_{\mathbb{Z}}, \mathscr{V}_{1}\right)$, and the action of $\square_{1}$ on $\{$ const. $\}$ is trivial. We omit the detail. Now, we can find solution to (1.7)-(1.8), whose proof is similar to that of Theorem 1.2 in [40] for the $k$-Cauchy-Fueter operator on $\mathbb{H}^{n}$.

Theorem 4.1 Suppose that $\operatorname{dim} \mathscr{H} \geq 19$ and $k=2,3, \ldots$ If $f \in \operatorname{Dom}\left(\mathscr{D}_{1}\right)$ is $\mathscr{D}_{1}$-closed and $f \perp$ constant vectors, then there exist $u \in L^{2}\left(\mathscr{H}_{/} / \mathscr{H}_{\mathbb{Z}}, \mathscr{V}_{0}\right)$ such that

$$
\mathscr{D}_{0} u=f .
$$

Proof The $L^{2}$ estimate (3.2) implies

$$
c_{n, k}\|g\|^{2} \leq\left\|\mathscr{D}_{0}^{*} g\right\|^{2}+\left\|\mathscr{D}_{1} g\right\|^{2}=\left\langle\square_{1} g, g\right\rangle \leq\left\|\square_{1} g\right\|\|g\|,
$$

for $g \in \operatorname{Dom}\left(\square_{1}\right)$, i.e.,

$$
c_{n, k}\|g\| \leq\left\|\square_{1} g\right\| .
$$


Thus, $\square_{1}: \operatorname{Dom}\left(\square_{1}\right) \rightarrow \mathcal{L}$ is injective. This together with the self-adjointness of $\square_{1}$ by Proposition 4.1 implies the density of the range. For fixed $f \in \mathcal{L}$, the complex anti-linear functional

$$
l_{f}: \square_{1} g \longrightarrow\langle f, g\rangle
$$

is then well-defined on a dense subspace of $\mathcal{L}$. It is finite since

$$
\left|l_{f}\left(\square_{1} g\right)\right|=|\langle f, g\rangle| \leq\|f\|\|g\| \leq \frac{1}{c_{n, k}}\|f\|\left\|\square_{1} g\right\|
$$

for any $g \in \operatorname{Dom}\left(\square_{1}\right)$, by (4.1). So $l_{f}$ can be uniquely extended to a continuous anti-linear functional on $\mathcal{L}$. By the Riesz representation theorem, there exists a unique element $h \in \mathcal{L}$ such that $l_{f}(F)=\langle h, F\rangle$ for any $F \in \mathcal{L}$, and $\|h\|=\left\|l_{f}\right\| \leq \frac{1}{c_{n, k}}\|f\|$. Then, we have

$$
\left\langle h, \square_{1} g\right\rangle=\langle f, g\rangle
$$

for any $g \in \operatorname{Dom}\left(\square_{1}\right)$. This implies that $h \in \operatorname{Dom}\left(\square_{1}^{*}\right)$ and $\square_{1}^{*} h=f$, and so $h \in \operatorname{Dom}\left(\square_{1}\right)$ and $\square_{1} h=f$ by self-adjointness of $\square_{1}$. We write $h=N f$. Then, $\|N f\| \leq \frac{1}{c_{n, k}}\|f\|$.

Since $N f \in \operatorname{Dom}\left(\square_{1}\right)$, we have $\mathscr{D}_{0}^{*} N f \in \operatorname{Dom}\left(\mathscr{D}_{0}\right), \mathscr{D}_{1} N f \in \operatorname{Dom}\left(\mathscr{D}_{1}^{*}\right)$, and

$$
\mathscr{D}_{0} \mathscr{D}_{0}^{*} N f=f-\mathscr{D}_{1}^{*} \mathscr{D}_{1} N f
$$

by $\square_{1} N f=f$. Because $f$ and $\mathscr{D}_{0} F$ for any $F \in \operatorname{Dom}\left(\mathscr{D}_{0}\right)$ are both $\mathscr{D}_{1}$-closed, the above identity implies $\mathscr{D}_{1}^{*} \mathscr{D}_{1} N f \in \operatorname{Dom}\left(\mathscr{D}_{1}\right)$ and so $\mathscr{D}_{1} \mathscr{D}_{1}^{*} \mathscr{D}_{1} N f=0$. Then,

$$
0=\left\langle\mathscr{D}_{1} \mathscr{D}_{1}^{*} \mathscr{D}_{1} N f, \mathscr{D}_{1} N f\right\rangle=\left\|\mathscr{D}_{1}^{*} \mathscr{D}_{1} N f\right\|^{2},
$$

i.e., $\mathscr{D}_{1}^{*} \mathscr{D}_{1} N f=0$. Hence, $\mathscr{D}_{0} \mathscr{D}_{0}^{*} N f=f$ by (4.2).

\subsection{Proof of Hartogs' phenomenon}

We need the analytic hypoellipticity of $\Delta_{b}$. Let $G$ be a nilpotent Lie group of step 2, and its Lie algebra $\mathfrak{g}$ has decomposition: $\mathfrak{g}=\mathfrak{g}_{1} \oplus \mathfrak{g}_{2}$ satisfying $\left[\mathfrak{g}_{1}, \mathfrak{g}_{1}\right] \subset \mathfrak{g}_{2},\left[\mathfrak{g}, \mathfrak{g}_{2}\right]=0$. Consider the condition $(H)$ : For any $\lambda \in \mathfrak{g}_{2}^{*} \backslash\{0\}$, the antisymmetric bilinear form

$$
B_{\lambda}\left(Y, Y^{\prime}\right)=\left\langle\lambda,\left[Y, Y^{\prime}\right]\right\rangle,
$$

for $Y, Y^{\prime} \in \mathfrak{g}_{1}$ is nondegenerate. Métivier proved the following theorem for analytic hypoellipticity.

Theorem 4.2 ([24, Theorem 0]) Let $P$ be a homogeneous left invariant differential operator on a nilpotent Lie group $G$ satisfies condition $(H)$. Then, the following are equivalent:

(i) $P$ is analytic hypoelliptic;

(ii) $P$ is $C^{\infty}$ hypoelliptic.

Corollary $4.1 \Delta_{b}$ is analytic hypoelliptic on a domain $\Omega \subset \mathscr{H}$, i.e., for any distribution $u \in S^{\prime}(\Omega)$ such that $\Delta_{b} u$ is analytic, $u$ must be also analytic.

Proof It follows from the well-known subellipticity of $\Delta_{b}$ that $u$ is locally $C^{k+1}$ if $\Delta_{b} u$ is locally $C^{k}$. So $\Delta_{b}$ is $C^{\infty}$ hypoelliptic. To obtain the analytic hypoellipticity of $\Delta_{b}$ by applying Theorem 4.2, it is sufficient to check the condition $(H)$ for the right quaternionic Heisenberg group $\mathscr{H}$. In this case, $\mathfrak{g}_{1}=\operatorname{span}\left\{Y_{1}, \ldots, Y_{4 n}\right\}, \mathfrak{g}_{2}=\operatorname{span}\left\{\partial_{s_{1}}, \partial_{s_{2}}, \partial_{s_{3}}\right\}$, where 
$Y_{1}, \ldots, Y_{4 n}$ is the left invariant vector fields in (2.4). Let $\lambda \in \mathfrak{g}_{2}^{*} \backslash\{0\}$. For $Y_{4 l+j}, Y_{4 l+j^{\prime}} \in \mathfrak{g}_{1}$, we have

$$
B_{\lambda}\left(Y_{4 l+j}, Y_{4 l^{\prime}+j^{\prime}}\right)=\left\langle\lambda,\left[Y_{4 l+j}, Y_{4 l^{\prime}+j^{\prime}}\right]\right\rangle=4 \delta_{l l^{\prime}} \sum_{\beta=1}^{3} B_{j j^{\prime}}^{\beta} \lambda\left(\partial_{s_{\beta}}\right)=4 \delta_{l l^{\prime}} \sum_{\beta=1}^{3} B_{j j^{\prime}}^{\beta} \lambda_{\beta},
$$

by (2.5), if we write $\lambda\left(\partial_{s_{\beta}}\right)=\lambda_{\beta}$. Then, the matrix associated with $B_{\lambda}$ is

$$
4 \sum_{\beta=1}^{3}\left(\begin{array}{ccc}
\lambda_{\beta} B^{\beta} & & \\
& \ddots & \\
& & \lambda_{\beta} B^{\beta}
\end{array}\right) \text {, where } \sum_{\beta=1}^{3} \lambda_{\beta} B^{\beta}=\left(\begin{array}{cccc}
0 & -\lambda_{1} & -\lambda_{2} & -\lambda_{3} \\
\lambda_{1} & 0 & -\lambda_{3} & \lambda_{2} \\
\lambda_{2} & \lambda_{3} & 0 & -\lambda_{1} \\
\lambda_{3} & -\lambda_{2} & \lambda_{1} & 0
\end{array}\right),
$$

whose determinant is $\left(\lambda_{1}^{2}+\lambda_{2}^{2}+\lambda_{3}^{2}\right)^{2 n}$ by direct calculation. So $B_{\lambda}$ is nondegenerate for $\lambda \in \mathfrak{g}_{2}^{*} \backslash\{0\}$, i.e., $\mathscr{H}$ satisfies condition $(H)$.

Liouville-type theorems hold for SubLaplacian $\Delta_{b}$ on the right quaternionic Heisenberg group by the following general theorem of Geller.

Theorem 4.3 ([14, Theorem 2]) Let $\mathscr{L}$ be a homogeneous hypoelliptic left invariant differential operator on a homogeneous group $G$. Suppose $u \in S^{\prime}(G)$ and $\mathscr{L} u=0$. Then, $u$ is a polynomial.

Theorem 4.4 Let $\widetilde{\Omega}$ be an open set in $\mathscr{F}$ such that $\widetilde{\Omega} \Subset \mathscr{F}$ and $\mathscr{F} \backslash \widetilde{\Omega}$ are connected. If $f \in C^{1}\left(\mathscr{H} / \mathscr{H}_{\mathbb{Z}}, \mathscr{V}_{1}\right)$ with supp $f \subset \widetilde{\Omega}$ is $\mathscr{D}_{1}$-closed and $f \perp$ constant vectors, then there exist $u \in C^{2}\left(\mathscr{H} / \mathscr{H}_{\mathbb{Z}}, \mathscr{V}_{0}\right)$ such that

$$
\mathscr{D}_{0} u=f,
$$

with $\operatorname{supp} u \subset \widetilde{\Omega}$.

Proof By Theorem 4.1, we can find a solution $u \in L^{2}\left(\mathscr{H} / \mathscr{H}_{\mathbb{Z}}, \mathscr{V}_{0}\right)$ to (4.4). For $c \in \mathbb{H}$, denote

$$
\mathscr{H}_{c}^{\prime}:=\left\{\left(q^{\prime}, c, s\right) \in \mathscr{H}: q^{\prime} \in \mathbb{H}^{n-1}, s \in \mathbb{R}^{3}\right\} .
$$

We see that $\mathscr{H}_{c}^{\prime} \cap \Omega=\emptyset$ for $|c|$ small by $\widetilde{\Omega} \Subset \mathscr{\mathscr { F }}$.

Since $\mathscr{D}_{0} u=0$ on $\left(\mathscr{H} / \mathscr{H}_{\mathbb{Z}}\right) \backslash \widetilde{\Omega}$, we have $\mathscr{D}_{0}^{*} \mathscr{D}_{0} u=0$, and then, by Proposition 2.4 $\Delta_{b} u_{A_{1}^{\prime} \ldots A_{k}^{\prime} A}=0$ on $\left(\mathscr{H} / \mathscr{H}_{\mathbb{Z}}\right) \backslash \widetilde{\Omega}$ in the sense of distributions for any fixed $A_{1}^{\prime}, \ldots, A_{k}^{\prime}, A$. So it is real analytic on $\left(\mathscr{H} / \mathscr{H}_{\mathbb{Z}}\right) \backslash \widetilde{\Omega}$ by Corollary 4.1 . Moreover, $u$ is $C^{2}$ on $\mathscr{H} / \mathscr{H}_{\mathbb{Z}}$ by subellipticity of $\Delta_{b}$. In particular, $u\left(q^{\prime}, c, s\right)$ is well-defined on $\mathscr{H}_{c}^{\prime} / \mathscr{H}_{\mathbb{Z}}^{\prime}$ as a real analytic function. So it can be extended to a periodic function over $\mathscr{H}_{c}^{\prime}$ by (2.11). Now, let $\mathscr{D}_{0}^{\prime}$ be the tangential $k$-Cauchy-Fueter operator on $\mathscr{H}_{c}^{\prime}$, i.e., $\mathscr{D}_{0}^{\prime} u$ is a $\odot^{k-1} \mathbb{C}^{2} \otimes \mathbb{C}^{2 n-2}$-valued function with

$$
\left(\mathscr{D}_{0}^{\prime} u\right)_{A A_{2}^{\prime} \ldots A_{k}^{\prime}}=\left(\mathscr{D}_{0} u\right)_{A A_{2}^{\prime} \ldots A_{k}^{\prime}}, \quad A=0,1, \ldots, 2 n-3 .
$$

By applying Proposition 2.4 to $\mathscr{H}_{c}^{\prime}$, we see that $\Delta_{b}^{\prime} u=0$, where $\Delta_{b}^{\prime}=-\sum_{a=0}^{4 n-5} Y_{a}^{2}$. Then, apply Liouville-type Theorem 4.3 to the group $\mathscr{H}_{c}^{\prime}$ and $\Delta_{b}^{\prime}$ to get

$$
u(\cdot, c, \cdot)=\text { a polynomial on } \mathscr{H}_{c}^{\prime},
$$

which must be a constant by periodicity. Thus, $u$ only depends on the variable $q_{n}$. 
Similarly, we can prove $u$ is a constant on the subgroup

$$
\mathscr{H}_{0}^{\prime \prime}:=\left\{\left(0, q_{n}, s\right) \in \mathscr{H} ; q_{n} \in \mathbb{H}, s \in \mathbb{R}^{3}\right\} .
$$

Now, if replacing $u$ by $u$ - const., we see that $u$ vanishes in a neighborhood of $\mathscr{H}_{0}^{\prime \prime}$. Consequently, by the identity theorem for real analytic functions it vanishes on the connected component $\mathscr{F} \backslash \widetilde{\Omega}$. Thus, supp $u \subset \widetilde{\Omega}$.

The solution with supp $u \subset \widetilde{\Omega}$ above plays the role of compactly supported solution to $\bar{\partial}$ equation or the tangential $k$-Cauchy-Fueter equations (cf., e.g., [17,35]). It leads to Hartogs' extension phenomenon as follows.

The proof of Theorem 1.1 Without loss of generality, we can assume $\Omega \Subset \mathscr{F}$ by dilating if necessary. Let $\chi \in C_{0}^{\infty}(\Omega)$ be equal to 1 in a neighborhood of $K$ such that $\mathscr{F} \backslash \operatorname{supp} \chi$ is connected. Set

$$
\tilde{u}(\xi):=\left\{\begin{array}{ll}
(1-\chi) u(\xi), & \xi \in \Omega \backslash K \\
0, & \xi \in K
\end{array} .\right.
$$

Then, $\tilde{u} \in C^{\infty}(\Omega)$, and $\left.\tilde{u}\right|_{\Omega \backslash \operatorname{supp} \chi}=\left.u\right|_{\Omega \backslash \operatorname{supp} \chi}$. We have

$$
\mathscr{D}_{0} \tilde{u}=\mathscr{D}_{0}((1-\chi) u)=: f
$$

on $\mathscr{H}$, where $f_{A_{2}^{\prime} \ldots A_{k}^{\prime} A}=-\sum_{A_{1}^{\prime}} Z_{A}^{A_{1}^{\prime}} \chi \cdot u_{A_{1}^{\prime} \ldots A_{k}^{\prime}}$ by $\mathscr{D}_{0} u=0$ on $\Omega \backslash K$. Hence, $f \in$ $C_{0}^{\infty}\left(\mathscr{H}, \mathscr{V}_{1}\right)$ vanishes in $K$ and outside $\Omega$, satisfying $\mathscr{D}_{1} f=\mathscr{D}_{1} \mathscr{D}_{0} \tilde{u}=0$ by (2.30). We can extend $f$ to a periodic function and view it as an element of $C^{\infty}\left(\mathscr{H} / \mathscr{H}_{\mathbb{Z}}, \mathscr{V}_{1}\right)$.

Denote

$$
c:=\frac{\int_{\mathscr{H} / \mathscr{H}_{\mathbb{Z}}} f \mathrm{~d} V}{\int_{\mathscr{H} / \mathscr{H}_{\mathbb{Z}}} \mathrm{d} V} \in \mathscr{V}_{1} .
$$

Then, we have $(f-c) \perp$ constant vectors. It follows from Theorem 4.4 that there exists a solution $\widetilde{U} \in C^{2}\left(\mathscr{H} / \mathscr{H}_{\mathbb{Z}}, \mathscr{V}_{0}\right)$ to $\mathscr{D}_{0} \widetilde{U}=f-c$, which vanishes outside $\widetilde{\Omega}:=\operatorname{supp} \chi$. Then, $\mathscr{D}_{0}(\tilde{u}-\widetilde{U})=c$ on $\mathscr{H} / \mathscr{H}_{\mathbb{Z}}$. So $c=\left.\mathscr{D}_{0} \widetilde{u}\right|_{\Omega \backslash \widetilde{\Omega}}=\left.\mathscr{D}_{0} u\right|_{\Omega \backslash \widetilde{\Omega}}=0$. Therefore, $U=\tilde{u}-\widetilde{U}$ is $k$-CF in $\Omega$ since $\mathscr{D}_{0}(\widetilde{u}-\widetilde{U})=0$. Note that $\widetilde{U} \equiv 0$ outside $\widetilde{\Omega}$ and $\mathscr{F} \backslash \widetilde{\Omega}$ is connected. So $U=u$ in $\Omega \backslash \widetilde{\Omega}$. Then, $U=u$ in $\Omega \backslash K$ by the identity theorem for real analytic functions. The theorem is proved.

\section{The restriction of the $k$-Cauchy-Fueter operator to the hypersurface $\mathcal{S}$}

\subsection{The nilpotent Lie groups of step two associated with quadratic hypersurfaces}

Let $\left(x_{1}, \ldots, x_{4 n}, t_{1}, t_{2}, t_{3}\right)$ be coordinates of $\mathbb{R}^{4 n+3}$. Now, consider general quadratic hypersurfaces $\widehat{\mathcal{S}}$ defined by

$$
\rho=\operatorname{Re} q_{n+1}-\phi\left(q^{\prime}\right), \quad \text { where } \phi=\sum_{k=1}^{4 n} \mathbb{S}_{j k} x_{j} x_{k},
$$

for some symmetric matrix $\mathbb{S}$. Define the projection:

$$
\begin{aligned}
& \pi: \quad \widehat{\mathcal{S}} \quad \longrightarrow \mathbb{H}^{n} \times \operatorname{Im} \mathbb{H} \simeq \mathbb{R}^{4 n+3}, \\
& \left(q_{1}, \ldots, q_{n}, \phi\left(q^{\prime}\right)+\mathbf{t}\right) \longmapsto\left(q_{1}, \ldots, q_{n}, \mathbf{t}\right),
\end{aligned}
$$


where $\mathbf{t}=t_{1} \mathbf{i}+t_{2} \mathbf{j}+t_{3} \mathbf{k}, q_{l+1}=x_{4 l+1}+\mathbf{i} x_{4 l+2}+\mathbf{j} x_{4 l+3}+\mathbf{k} x_{4 l+4}, l=0, \ldots, n-1$ and $t_{\beta}=x_{4 n+1+\beta}$ for $\beta=1,2,3$. Let $\psi: \mathbb{H}^{n} \times \operatorname{Im} \mathbb{H} \longrightarrow \mathcal{S} \subset \mathbb{H}^{n+1}$ be its inverse. The Cauchy-Fueter operator is

$$
\bar{\partial}_{q_{l+1}}=\partial_{x_{4 l+1}}+\mathbf{i} \partial_{x_{4 l+2}}+\mathbf{j} \partial_{x_{4 l+3}}+\mathbf{k} \partial_{x_{4 l+4}} .
$$

Then, $\bar{\partial}_{q_{l+1}}+\bar{\partial}_{q_{l+1}} \phi \cdot \bar{\partial}_{q_{n+1}}$ is a vector field tangential to the hypersurface $\widehat{\mathcal{S}}$, since

$$
\left(\bar{\partial}_{q_{l+1}}+\bar{\partial}_{q_{l+1}} \phi \cdot \bar{\partial}_{q_{n+1}}\right) \rho=0,
$$

$l=0,1, \ldots, n-1$. This vector field is exactly the pushforward vector field $\psi_{*}\left(\bar{\partial}_{q_{l+1}}+\right.$ $\left.\bar{\partial}_{q_{l+1}} \phi \cdot \bar{\partial}_{\mathbf{t}}\right)$, where $\bar{\partial}_{\mathbf{t}}=\mathbf{i} \partial_{t_{1}}+\mathbf{j} \partial_{t_{2}}+\mathbf{k} \partial_{t_{3}}$. Because

$$
\psi_{*} \partial_{t_{\beta}}=\partial_{x_{4 n+1+\beta}}, \quad \psi_{*} \partial_{x_{4 l+j}}=\partial_{x_{4 l+j}}+\partial_{x_{4 l+j}} \phi \cdot \partial_{x_{4 n+1}},
$$

for $\beta=1,2,3, j=1, \ldots, 4, l=0, \ldots, n-1$, and

$$
\begin{aligned}
\psi_{*}\left(\bar{\partial}_{q_{l+1}}+\bar{\partial}_{q_{l+1}} \phi \cdot \bar{\partial}_{\mathbf{t}}\right)= & \sum_{j=1}^{4} \mathbf{i}_{j-1}\left(\partial_{x_{4 l+j}}+\partial_{x_{4 l+j}} \phi \cdot \partial_{x_{4 n+1}}\right) \\
& +\bar{\partial}_{q_{l+1}} \phi\left(\mathbf{i} \partial_{x_{4 n+2}}+\mathbf{j} \partial_{x_{4 n+3}}+\mathbf{k} \partial_{x_{4 n+4}}\right)=\bar{\partial}_{q_{l+1}}+\bar{\partial}_{q_{l+1}} \phi \cdot \bar{\partial}_{q_{n+1}} .
\end{aligned}
$$

Denote

$$
X_{4 l+1}+\mathbf{i} X_{4 l+2}+\mathbf{j} X_{4 l+3}+\mathbf{k} X_{4 l+4}:=\bar{\partial}_{q_{l+1}}+\bar{\partial}_{q_{l+1}} \phi \cdot \bar{\partial}_{\mathbf{t}} .
$$

Proposition 5.1 We have

$$
X_{b}=\partial_{x_{b}}+2 \sum_{\beta=1}^{3} \sum_{a=1}^{4 n}\left(\mathbb{S I}^{\beta}\right)_{a b} x_{a} \partial_{t_{\beta}},
$$

where $\mathbb{I}^{\beta}$ is the $(4 n) \times(4 n)$ matrix $\operatorname{diag}\left(I^{\beta}, \ldots, I^{\beta}\right)$.

Proof The proof is similar to that of Proposition 2.1 in [39]. Consider right multiplication by $\mathbf{i}_{\beta}$. Note that

$$
\begin{aligned}
\left(x_{1}+x_{2} \mathbf{i}+x_{3} \mathbf{j}+x_{4} \mathbf{k}\right) \mathbf{i} & =-x_{2}+x_{1} \mathbf{i}+x_{4} \mathbf{j}-x_{3} \mathbf{k}, \\
\left(x_{1}+x_{2} \mathbf{i}+x_{3} \mathbf{j}+x_{4} \mathbf{k}\right) \mathbf{j} & =-x_{3}-x_{4} \mathbf{i}+x_{1} \mathbf{j}+x_{2} \mathbf{k}, \\
\left(x_{1}+x_{2} \mathbf{i}+x_{3} \mathbf{j}+x_{4} \mathbf{k}\right) \mathbf{k} & =-x_{4}+x_{3} \mathbf{i}-x_{2} \mathbf{j}+x_{1} \mathbf{k},
\end{aligned}
$$

we can write

$$
\begin{aligned}
\left(x_{1}+x_{2} \mathbf{i}+x_{3} \mathbf{j}+x_{4} \mathbf{k}\right) \mathbf{i}_{\beta} & =-\left(I^{\beta} x\right)_{1}-\left(I^{\beta} x\right)_{2} \mathbf{i}-\left(I^{\beta} x\right)_{3} \mathbf{j}-\left(I^{\beta} x\right)_{4} \mathbf{k} \\
& =-\sum_{j=1}^{4}\left(I^{\beta} x\right)_{j} \mathbf{i}_{j-1},
\end{aligned}
$$

where $I^{\beta}$,s are given by (2.41). $B^{\beta}$ in (2.2) is the matrix associated with left multiplication by $\mathbf{i}_{\beta}$ ([39, p. 1358]). Then, we have

$$
\begin{aligned}
\bar{\partial}_{q_{l+1}} \phi \cdot \partial_{\mathbf{t}} & =\left(\partial_{x_{4 l+1}} \phi+\mathbf{i} \partial_{x_{4 l+2}} \phi+\mathbf{j} \partial_{x_{4 l+3}} \phi+\mathbf{k} \partial_{x_{4 l+4}} \phi\right)\left(\mathbf{i} \partial_{t_{1}}+\mathbf{j} \partial_{t_{2}}+\mathbf{k} \partial_{t_{3}}\right) \\
& =-\sum_{\beta=1}^{3} \sum_{j, k=1}^{4} I_{j k}^{\beta} \partial_{x_{4 l+k}} \phi \mathbf{i}_{j-1} \partial_{t_{\beta}} .
\end{aligned}
$$


Substitute it into (5.3) to get

$$
X_{4 l+j}=\partial_{x_{4 l+j}}+2 \sum_{\beta=1}^{3} \sum_{k=1}^{4} \sum_{a=1}^{4 n} I_{k j}^{\beta} \mathbb{S}_{a(4 l+k)} x_{a} \partial_{t_{\beta}}=\partial_{x_{4 l+j}}+2 \sum_{\beta=1}^{3} \sum_{a=1}^{4 n}\left(\mathbb{S I}^{\beta}\right)_{a(4 l+j)} x_{a} \partial_{t_{\beta}},
$$

by the antisymmetry of $I^{\beta}$.

By Proposition 5.1, we get

$$
\left[X_{a}, X_{b}\right]=2 \sum_{\beta=1}^{3}\left(\left(\mathbb{S I}^{\beta}\right)_{a b}-\left(\mathbb{S I}^{\beta}\right)_{b a}\right) \partial_{t_{\beta}} .
$$

So $\operatorname{span}_{\mathbb{C}}\left\{X_{1}, \ldots, X_{4 n}, \partial_{t_{1}}, \partial_{t_{2}}, \partial_{t_{3}}\right\}$ is a nilpotent Lie algebra with center $\operatorname{span}_{\mathbb{C}}\left\{\partial_{t_{1}}, \partial_{t_{2}}\right.$, $\left.\partial_{t_{3}}\right\}$. The corresponding nilpotent Lie group of step two is the group associated with the quadratic hypersurface $\widehat{\mathcal{S}}$.

Now, if we choose the matrix $\mathbb{S}$ so that

$$
\mathbb{S I}^{\beta}+\mathbb{I}^{\beta} \mathbb{S}=2 \mathbb{B}^{\beta}, \quad \text { where } \mathbb{B}^{\beta}=\operatorname{diag}\left(B^{\beta}, \ldots, B^{\beta}\right),
$$

then the Lie algebra spanned by $X_{1}, \ldots, X_{4 n}, \partial_{t_{1}}, \partial_{t_{1}}, \partial_{t_{3}}$ is isomorphic to the Lie algebra of the right quaternionic Heisenberg group $\mathscr{H}$. It is sufficient to choose $\mathbb{S}=\operatorname{diag}(S, \ldots, S)$ such that $S I^{\beta}+I^{\beta} S=2 B^{\beta}$, where $S$ is a symmetric $4 \times 4$ matrix. Namely,

$$
C^{\beta}-\left(C^{\beta}\right)^{t}=2 B^{\beta},
$$

for $C^{\beta}=S I^{\beta}$. Then,

$$
S=\operatorname{diag}(-3,1,1,1)
$$

and

$$
\begin{aligned}
C^{1} & :=\left(\begin{array}{cccc}
0 & -3 & 0 & 0 \\
-1 & 0 & 0 & 0 \\
0 & 0 & 0 & -1 \\
0 & 0 & 1 & 0
\end{array}\right), C^{2}:=\left(\begin{array}{cccc}
0 & 0 & -3 & 0 \\
0 & 0 & 0 & 1 \\
-1 & 0 & 0 & 0 \\
0 & -1 & 0 & 0
\end{array}\right), \\
C^{3} & :=\left(\begin{array}{cccc}
0 & 0 & 0 & -3 \\
0 & 0 & -1 & 0 \\
0 & 1 & 0 & 0 \\
-1 & 0 & 0 & 0
\end{array}\right) .
\end{aligned}
$$

satisfy (5.5). Thus, the defining function (5.1) of $\widehat{\mathcal{S}}$ in this case is (1.2) of $\mathcal{S}$, and so the Lie group associate with $\mathcal{S}$ is the right quaternionic Heisenberg group.

\subsection{The restriction of the $k$-Cauchy-Fueter operator}

$X_{a}$ 's for $\mathcal{S}$ has the form

$$
X_{4 l+j}=\partial_{x_{4 l+j}}+2 \sum_{\beta=1}^{3} \sum_{k=1}^{4} C_{k j} x_{4 l+k} \partial_{t_{\beta}} .
$$

Since $C^{\beta}$ is not antisymmetric, they are different from the standard left invariant vector fields (2.4) on $\mathscr{H}$. It is standard that they can be transformed to the standard left invariant vector 
fields (2.4) on $\mathscr{H}$ by a simple coordinate transformation $\mathcal{F}: \mathscr{H} \rightarrow \mathbb{R}^{4 n+3},(y, s) \mapsto(x, t)$ given by

$$
x_{4 l+j}=y_{4 l+j}, \quad t_{\beta}=s_{\beta}+\sum_{k, j=1}^{4} D_{k j}^{\beta} y_{4 l+k} y_{4 l+j},
$$

(cf. [39, (1.8)]) with $D^{\beta}:=C^{\beta}+\left(C^{\beta}\right)^{t}$ symmetric. It is direct to see that

$$
\mathcal{F}_{*} \partial_{s_{\beta}}=\partial_{t_{\beta}} \quad \text { and } \quad \mathcal{F}_{*} Y_{4 l+j}=X_{4 l+j},
$$

where $Y_{4 l+j}$ is given by (2.4). Then, we find the relationship between complex horizontal vector fields $Z_{A}^{A^{\prime}}$,s on $\mathscr{H}$ and $\nabla_{A}^{A^{\prime}}$,s on $\mathbb{H}^{n+1}$.

Proposition 5.2 Under the diffeomorphism $\psi \circ \mathcal{F}: \mathscr{H} \rightarrow \mathcal{S}$, we have

$$
(\psi \circ \mathcal{F})_{*} Z_{A}^{A^{\prime}}=\nabla_{A}^{A^{\prime}}+\sum_{\alpha=0,1} C_{A}^{\alpha} \nabla_{(2 n+\alpha)}^{A^{\prime}}, \quad \text { for }\left(C_{A}^{\alpha}\right):=\left(\begin{array}{c}
\vdots \\
\tau\left(\bar{\partial}_{q_{l}} \phi\right) \\
\vdots
\end{array}\right),
$$

for fixed $A=0,1, \ldots, 2 n-1, A^{\prime}=0^{\prime}, 1^{\prime}$, where $\tau$ is the embedding given by (2.13).

Proof As $\tau$ is a representation, we have

$$
\begin{aligned}
\psi_{*} & \left(\begin{array}{rr}
-X_{4 l+3}-\mathbf{i} X_{4 l+4}-X_{4 l+1}-\mathbf{i} X_{4 l+2} \\
X_{4 l+1}-\mathbf{i} X_{4 l+2} & -X_{4 l+3}+\mathbf{i} X_{4 l+4}
\end{array}\right)=\psi_{*}\left(\begin{array}{cc}
X_{4 l+1}+\mathbf{i} X_{4 l+2}-X_{4 l+3}-\mathbf{i} X_{4 l+4} \\
X_{4 l+3}-\mathbf{i} X_{4 l+4} & X_{4 l+1}-\mathbf{i} X_{4 l+2}
\end{array}\right) \varepsilon \\
= & \tau\left(\psi_{*}\left(X_{4 l+1}+\mathbf{i} X_{4 l+2}+\mathbf{j} X_{4 l+3}+\mathbf{k} X_{4 l+4}\right)\right) \varepsilon \\
= & \tau\left(\bar{\partial}_{q_{l+1}}+\bar{\partial}_{q_{l+1}} \phi \cdot \bar{\partial}_{q_{n+1}}\right) \varepsilon=\tau\left(\bar{\partial}_{q_{l+1}}\right) \varepsilon+\tau\left(\bar{\partial}_{q_{l+1}} \phi\right) \tau\left(\bar{\partial}_{q_{n+1}}\right) \varepsilon \\
& =\left(\begin{array}{cc}
\nabla_{(2 l)}^{0^{\prime}} & \nabla_{((2 l)}^{1^{\prime}} \\
\nabla_{(2 l+1)}^{0^{\prime}} & \nabla_{(2 l+1)}^{1^{\prime}}
\end{array}\right)+\tau\left(\bar{\partial}_{q_{l}} \phi\right)\left(\begin{array}{cc}
\nabla_{(2 n)}^{0^{\prime}} & \nabla_{(2 n)}^{1^{\prime}} \\
\nabla_{(2 n+1)}^{0^{\prime}} & \nabla_{(2 n+1)}^{1^{\prime}}
\end{array}\right),
\end{aligned}
$$

where $\varepsilon=\left(\begin{array}{cc}0 & -1 \\ 1 & 0\end{array}\right)$ in (2.15). Then, (5.7) follows.

From this proposition, we can derive the relationship between operators in $k$-Cauchy-Fueter complex on $\mathbb{H}^{n+1}$ and that in the tangential $k$-Cauchy-Fueter complex on $\mathscr{H}$.

Proposition 5.3 Suppose that $f$ is a k-regular function near $q_{0} \in \mathcal{S}$. Then, $(\psi \circ \mathcal{F})^{*} f$ is $k-C F$ on $\mathscr{H}$ near the point $\mathcal{F}^{-1}\left(\pi\left(q_{0}\right)\right)$.

Proof As $f$ is a $k$-regular function near $q_{0} \in \mathcal{S} \subset \mathbb{H}^{n+1}$, we have $\sum_{B^{\prime}=0^{\prime}, 1^{\prime}} \nabla_{A}^{B^{\prime}} f_{B^{\prime} A_{2}^{\prime} \ldots A_{k}^{\prime}}=$ 0 for any fixed $A=0,1, \ldots, 2 n+1, A_{2}^{\prime}, \ldots, A_{k}^{\prime}=0^{\prime}, 1^{\prime}$. Then, we find that

$$
\begin{aligned}
\left.\left(\mathscr{D}_{0}(\psi \circ \mathcal{F})^{*} f\right)_{A A_{2}^{\prime} \ldots A_{k}^{\prime}}\right|_{\mathcal{F}^{-1}\left(\pi\left(q_{0}\right)\right)} & \left.\sum_{B^{\prime}=0^{\prime}, 1^{\prime}} Z_{A}^{B^{\prime}}\left((\psi \circ \mathcal{F})^{*} f\right)_{B^{\prime} A_{2}^{\prime} \ldots A_{k}^{\prime}}\right|_{\mathcal{F}^{-1}\left(\pi\left(q_{0}\right)\right)} \\
& =\sum_{B^{\prime}=0^{\prime}, 1^{\prime}}(\psi \circ \mathcal{F})_{*} Z_{A}^{B^{\prime}} f_{B^{\prime} A_{2}^{\prime} \ldots A_{k}^{\prime}}\left(q_{0}\right) \\
& =\sum_{B^{\prime}}\left(\nabla_{A}^{B^{\prime}}+\sum_{\alpha=0,1} C_{A}^{\alpha} \nabla_{(2 n+\alpha)}^{B^{\prime}}\right) f_{B^{\prime} A_{2}^{\prime} \ldots A_{k}^{\prime}\left(q_{0}\right)=0,}
\end{aligned}
$$

for any fixed $A=0,1, \ldots, 2 n-1, A_{2}^{\prime}, \ldots, A_{k}^{\prime}=0^{\prime}, 1^{\prime}$, by Proposition 5.2. The proposition is proved. 


\section{Appendix}

In the case $n=2, k=2$, We have isomorphisms

$$
\odot^{2} \mathbb{C}^{2} \cong \mathbb{C}^{3}, \quad \mathbb{C}^{2} \otimes \mathbb{C}^{4} \cong \mathbb{C}^{8},
$$

by identifying $f \in \odot^{2} \mathbb{C}^{2}$ and $F \in \mathbb{C}^{2} \otimes \mathbb{C}^{4}$ with

$$
f:=\left(\begin{array}{c}
f_{0^{\prime} 0^{\prime}} \\
f_{0^{\prime} 1^{\prime}} \\
f_{1^{\prime} 1^{\prime}}
\end{array}\right), \quad F:=\left(\begin{array}{c}
F_{0^{\prime} 0} \\
\vdots \\
F_{0^{\prime} 3} \\
F_{1^{\prime} 0} \\
\vdots \\
F_{1^{\prime} 3}
\end{array}\right),
$$

respectively. The operator $\mathscr{D}_{0}$ in $(2.18)$ can be written as a $8 \times 3$ matrix-valued differential operator:

$$
\mathscr{D}_{0}=\left(\begin{array}{ccc}
-Y_{3}-\mathbf{i} Y_{4} & -Y_{1}-\mathbf{i} Y_{2} & 0 \\
Y_{1}-\mathbf{i} Y_{2} & -Y_{3}+\mathbf{i} Y_{4} & 0 \\
-Y_{7}-\mathbf{i} Y_{8} & -Y_{5}-\mathbf{i} Y_{6} & 0 \\
Y_{5}-\mathbf{i} Y_{6} & -Y_{7}+\mathbf{i} Y_{8} & 0 \\
0 & -Y_{3}-\mathbf{i} Y_{4} & -Y_{1}-\mathbf{i} Y_{2} \\
0 & Y_{1}-\mathbf{i} Y_{2} & -Y_{3}+\mathbf{i} Y_{4} \\
0 & -Y_{7}-\mathbf{i} Y_{8} & -Y_{5}-\mathbf{i} Y_{6} \\
0 & Y_{5}-\mathbf{i} Y_{6} & -Y_{7}+\mathbf{i} Y_{8}
\end{array}\right) .
$$

Similarly, the operator $\mathscr{D}_{1}$ in $(2.23)$ can be written as a $6 \times 8$ matrix-valued differential operator:

$$
\left(\begin{array}{cccccccc}
-Y_{1}+\mathbf{i} Y_{2} & -Y_{3}-\mathbf{i} Y_{4} & 0 & 0 & Y_{3}-\mathbf{i} Y_{4} & -Y_{1}-\mathbf{i} Y_{2} & 0 & 0 \\
Y_{7}+\mathbf{i} Y_{8} & 0 & -Y_{3}-\mathbf{i} Y_{4} & 0 & Y_{5}+\mathbf{i} Y_{6} & 0 & -Y_{1}-\mathbf{i} Y_{2} & 0 \\
-Y_{5}-\mathbf{i} Y_{6} & 0 & 0 & -Y_{3}-\mathbf{i} Y_{4} & Y_{7}-\mathbf{i} Y_{8} & 0 & 0 & -Y_{1}-\mathbf{i} Y_{2} \\
0 & Y_{7}+\mathbf{i} Y_{8} & Y_{1}-\mathbf{i} Y_{2} & 0 & 0 & Y_{5}+\mathbf{i} Y_{6} & -Y_{3}+\mathbf{i} Y_{4} & 0 \\
0 & -Y_{5}+\mathbf{i} Y_{6} & 0 & Y_{1}-\mathbf{i} Y_{2} & 0 & Y_{7}-\mathbf{i} Y_{8} & 0 & -Y_{3}+\mathbf{i} Y_{4} \\
0 & 0 & -Y_{5}+\mathbf{i} Y_{6} & -Y_{7}-\mathbf{i} Y_{8} & 0 & 0 & Y_{7}-\mathbf{i} Y_{8} & -Y_{5}-\mathbf{i} Y_{6}
\end{array}\right) .
$$

Thus, we have $\mathscr{D}_{0}^{*}=-{\overline{\mathscr{D}_{0}}}^{t}, \mathscr{D}_{1}^{*}=-\overline{\mathscr{D}}_{1}^{t}$. Then, by direct calculation we have

$$
\square_{1}=\mathscr{D}_{0} \mathscr{D}_{0}^{*}+\mathscr{D}_{1}^{*} \mathscr{D}_{1}=\left(\begin{array}{cc}
A & 0 \\
0 & B
\end{array}\right)
$$

with

$$
\begin{gathered}
A=\left(\begin{array}{cccc}
\Delta_{b}+\Delta_{1}-12 \mathbf{i} \partial_{s_{1}} & L_{1}+\left(Y_{1}+\mathbf{i} Y_{2}\right)\left(-Y_{3}-\mathbf{i} Y_{4}\right) & \left(-Y_{1}-\mathbf{i} Y_{2}\right)\left(Y_{5}-\mathbf{i} Y_{6}\right) & \left(-Y_{1}-\mathbf{i} Y_{2}\right)\left(Y_{7}+\mathbf{i} Y_{8}\right) \\
-\overline{L_{1}}+\left(Y_{3}-\mathbf{i} Y_{4}\right)\left(-Y_{1}+\mathbf{i} Y_{2}\right) & \Delta_{b}+\Delta_{2}+12 \mathbf{i} \partial_{s_{1}} & \left(-Y_{3}+\mathbf{i} Y_{4}\right)\left(Y_{5}-\mathbf{i} Y_{6}\right) & \left(-Y_{3}+\mathbf{i} Y_{4}\right)\left(Y_{7}+\mathbf{i} Y_{8}\right) \\
\left(-Y_{5}-\mathbf{i} Y_{6}\right)\left(Y_{1}-\mathbf{i} Y_{2}\right) & \left(-Y_{5}-\mathbf{i} Y_{6}\right)\left(Y_{3}+\mathbf{i} Y_{4}\right) & \Delta_{b}+\Delta_{3}-12 \mathbf{i} \partial_{s_{1}} & L_{1}+\left(Y_{5}+\mathbf{i} Y_{6}\right)\left(-Y_{7}-\mathbf{i} Y_{8}\right) \\
\left(-Y_{7}+\mathbf{i} \mathbf{Y}_{8}\right)\left(Y_{1}-\mathbf{i} Y_{2}\right) & \left(-Y_{7}+\mathbf{i} Y_{8}\right)\left(Y_{3}+\mathbf{i} Y_{4}\right) & -\overline{L_{1}}+\left(Y_{7}-\mathbf{i} Y_{8}\right)\left(-Y_{5}+\mathbf{i} Y_{6}\right) & \Delta_{b}+\Delta_{4}+12 \mathbf{i} \partial_{s_{1}}
\end{array}\right), \\
B=\left(\begin{array}{cccc}
\Delta_{b}+\Delta_{2}-12 \mathbf{i} \partial_{s_{1}} & L_{1}+\left(-Y_{3}-\mathbf{i} Y_{4}\right)\left(-Y_{1}-\mathbf{i} Y_{2}\right) & \left(-Y_{3}-\mathbf{i} Y_{4}\right)\left(-Y_{7}-\mathbf{i} Y_{8}\right) & \left(-Y_{3}-\mathbf{i} Y_{4}\right)\left(-Y_{5}-\mathbf{i} Y_{6}\right) \\
-\overline{L_{1}}+\left(Y_{1}-\mathbf{i} Y_{2}\right)\left(Y_{3}-\mathbf{i} Y_{4}\right) & \Delta_{b}+\Delta_{1}+12 \mathbf{i} \partial_{s_{1}} & \left(Y_{1}-\mathbf{i} Y_{2}\right)\left(Y_{7}-\mathbf{i} Y_{8}\right) & \left(Y_{1}-\mathbf{i} Y_{2}\right)\left(-Y_{5}-\mathbf{i} Y_{6}\right) \\
\left(Y_{7}-\mathbf{i} Y_{8}\right)\left(Y_{3}-\mathbf{i} Y_{4}\right) & \left(-Y_{7}-\mathbf{i} Y_{8}\right)\left(-Y_{1}-\mathbf{i} Y_{2}\right) & \Delta_{b}+\Delta_{4}-12 \mathbf{i} \partial_{s_{1}} & L_{1}+\left(-Y_{7}-\mathbf{i} Y_{8}\right)\left(-Y_{5}-\mathbf{i} Y_{6}\right) \\
\left(Y_{5}-\mathbf{i} Y_{6}\right)\left(Y_{3}-\mathbf{i} Y_{4}\right) & \left(Y_{5}-\mathbf{i} Y_{6}\right)\left(-Y_{1}-\mathbf{i} Y_{2}\right) & -\bar{L}_{1}+\left(Y_{5}-\mathbf{i} Y_{6}\right)\left(Y_{7}-\mathbf{i} Y_{8}\right) & \Delta_{b}+\Delta_{3}+12 \mathbf{i} \partial_{s_{1}}
\end{array}\right),
\end{gathered}
$$

where $\Delta_{b}=-Y_{1}^{2} \cdots-Y_{8}^{2}, \Delta_{1}=-Y_{1}^{2}-Y_{2}^{2}, \Delta_{2}=-Y_{3}^{2}-Y_{4}^{2}, \Delta_{3}=-Y_{5}^{2}-Y_{6}^{2}, \Delta_{4}=$ $-Y_{7}^{2}-Y_{8}^{2}, L_{1}=8\left(\partial_{s_{2}}+\mathbf{i} \partial_{s_{3}}\right)$. Because of the complexity of $\square_{1}$ in (6.3), it is not easy to obtain its fundamental solution. 


\section{References}

1. Adams, W., Loustaunau, P., Palamodov, V., Struppa, D.: Hartogs' phenomenon for polyregular functions and projective dimension of related modules over a polynomial ring. Ann. Inst. Fourier 47, 623-640 (1997)

2. Airapetjan, A., Henkin, M.: Integral representation of differential forms on Cauchy-Riemann manifolds and the theory of CR function. Russ. Math. Surv. 39, 41-118 (1984)

3. Andreotti, A., Hill, C.D., Lojasiewicz, S., Mackichan, B.: Complexes of differential operators. Invent. Math. 35(1), 43-86 (1976)

4. Andreotti, A., Nacinovich, M.: Noncharacteristic hypersurfaces for complexes of differential operators. Ann. Mat. Pura Appl. 125(1), 13-83 (1980)

5. Baston, R.: Quaternionic complexes. J. Geom. Phys. 8, 29-52 (1992)

6. Baston, R., Eastwood, M.: The Penrose Transform, its Interaction with Representation Theory, Oxford Mathematical Monographs. The Clarendon Press, New York (1989)

7. Brinkschulte, J., Hill, C.D., Nacinovich, M.: On the nonvanishing of abstract Cauchy-Riemann cohomology groups. Math. Ann. 363(1-2), 1-15 (2015)

8. Bureš, J., Souček, V.V.: Complexes of invariant differential operators in several quaternionic variables. Complex Var. Elliptic Equ. 51(5-6), 463-487 (2006)

9. Bureš, J., Damiano, A., Sabadini, I.: Explicit resolutions for several Fueter operators. J. Geom. Phys. 57, 765-775 (2007)

10. Chen, S.-C., Shaw, M.-C.: Partial Differential Equations in Several Complex Variables, AMS/IP Studies in Advanced Mathematics, vol. 19. American Mathematical Society, Providence (2001)

11. Colombo, F., Souček, V., Struppa, D.: Invariant resolutions for several Fueter operators. J. Geom. Phys. 56(7), 1175-1191 (2006)

12. Colombo, F., Sabadini, I., Sommen, F., Struppa, D.: Analysis of Dirac Systems and Computational Algebra, Progress in Mathematical Physics, vol. 39. Birkhäuser, Boston (2004)

13. Folland, G.B., Stein, E.M.: Estimates for the $\bar{\partial}_{b}$ complex and analysis on the Heisenberg group. Commun. Pure Appl. Math. 27(4), 429-522 (1974)

14. Geller, D.: Liouville's theorem for homogeneous groups. Commun. Partial Differ. Equ. 8 (15), 1621-1664 (1983)

15. Henkin, M.: The method of integral representations in complex analysis. In: Introduction to Complex Analysis, pp. 19-116. Springer, Berlin (1997)

16. Hill, C.D., Nacinovich, M.: On the failure of the Poincaré Lemma for $\bar{\partial}_{M}$ II. Math. Ann. 335(1), 193-219 (2006)

17. Hömander, L.: An Introduction to Complex Analysis in Several Variables, Second Revised Edition, North-Holland Mathematical Library, vol. 7. North-Holland Publishing Co, Amsterdam (1973)

18. Ivanov, S., Vassilev, D.: Extremals for the Sobolev Inequality and the Quaternionic Contact Yamabe Problem. World Scientific, Singopore (2011)

19. Ivanov, S., Minchev, I., Vassilev, D.: Quaternionic Contact Einstein Structures and the Quaternionic Contact Yamabe Problem, vol. 231. American Mathematical Society, Providence (2014)

20. Jerison, D.: The Poincaré inequality for vector fields satisfying Hörmander's condition. Duke Math. J. 53(2), 503-523 (1986)

21. Kang, Q.-Q., Wang, W.: On Penrose integral formula and series expansion of $k$-regular functions on the quaternionic space $\mathbb{H}^{n}$. J. Geom. Phys. 64(1), 192-208 (2013)

22. Kobayashi, S., Nomizu, K.: Foundations of Differential Geometry. Interscience Publishers, New York (1963)

23. Laurent-Thiébaut, C., Leiterer, J.: On the Hartogs-Bochner extension phenomenon for differential forms. Math. Ann. 284(1), 103-119 (1989)

24. Métivier, G.: Hypoellipticité analytique sur des groupes nilpotents de rang 2. Duke Math. J. 47(1), 195-221 (1980)

25. Nacinovich, M.: Complex Analysis and Complexes of Differential Operators, Springer Lecture Notes in Mathematics, vol. 287, pp. 105-195. Springer, Berlin (1973)

26. Palamodov, V.: Hartogs phenomenon for systems of differential equations. J. Geom. Anal. 24(2), 667-686 (2014)

27. Pandžić, P., Souček, V.: BGG complexes in singular infinitesimal character for type A. J. Math. Phys. 58, $111512(2017)$

28. Pertici, D.: Funzioni regolari di piu variabili quaternioniche. Ann. Mat. Pura Appl. 151, 39-65 (1988)

29. Porten, E.: The Hartogs phenomenon on weakly pseudoconcave hypersurfaces. Math. Ann. 354(2), 659683 (2012) 
30. Sabadini, I., Sommen, F., Struppa, D.C., Van Lancker, P.: Complexes of Dirac operators in Clifford algebras. Math. Z. 239, 293-320 (2002)

31. Shi, Y., Wang, W.: On conformal qc geometry, spherical qc manifolds and convex cocompact subgroups of $\operatorname{Sp}(n+1,1)$. Ann. Glob. Anal. Geom. 49(3), 271-307 (2016)

32. Shi, Y., Wang, W.: The Szegö kernel for $k$-CF functions on the quaternionic Heisenberg group. Appl. Anal. 96(14), 2474-2492 (2017)

33. Wan, D.-R., Wang, W.: On the quaternionic Monge-Ampère operator, closed positive currents and LelongJensen type formula on the quaternionic space. Bull. Sci. Math. 141(4), 267-311 (2017)

34. Wang, H.-Y., Ren, G.-B.: Bochner-Martinelli formula for $k$-Cauchy-Fueter operator. J. Geom. Phys. 84, 43-54 (2014)

35. Wang, W.: The $k$-Cauchy-Fueter complex, Penrose transformation and Hartogs' phenomenon for quaternionic $k$-regular functions. J. Geom. Phys. 60(3), 513-530 (2010)

36. Wang, W.: The Yamabe problem on quaternionic contact manifolds. Ann. Mat. Pura Appl. 186, 359-380 (2007)

37. Wang, W.: The tangential Cauchy-Fueter complex on the quaternionic Heisenberg group. J. Geom. Phys. 61(1), 363-380 (2011)

38. Wang, W.: On the optimal control method in quaternionic analysis. Bull. Sci. Math. 135(8), 988-1010 (2011)

39. Wang, W.: On the tangential Cauchy-Fueter operators on nondegenerate quadratic hypersurfaces in $\mathbb{H}^{2}$. Math. Nach. 286(13), 1353-1376 (2013)

40. Wang, W.: On the weighted $L^{2}$ estimate for the $k$-Cauchy-Fueter operator and the weighted $k$-Bergman kernel. J. Math. Anal. Appl. 452(1), 685-707 (2017)

41. Wang, W.: On quaternionic complexes over unimodular quaternionic manifolds. Differ. Geom. Appl. 58, 227-253 (2018)

42. Wang, W.: The Neumann problem for the $k$-Cauchy-Fueter complex over $k$-pseudoconvex domains in $\mathbb{R}^{4}$ and the $L^{2}$ Estimate. J. Geom. Anal. 29, 1233-1258 (2019)

Publisher's Note Springer Nature remains neutral with regard to jurisdictional claims in published maps and institutional affiliations. 